\title{
MOLECULAR DYNAMICS AT AN ENERGY-LEVEL CROSSING
}

\author{
PHILIPPE BRIET $^{1} \&$ ANDRÉ MARTINEZ ${ }^{2}$
}

\begin{abstract}
This paper is a continuation of a previous work BrMa about the study of the survival probability modelizing the molecular predissociation in the Born-Oppenheimer framework. Here we consider the critical case where the reference energy corresponds to the value of a crossing of two electronic levels, one of these two levels being confining while the second dissociates. We show that the survival probability associated to a certain initial state is a sum of the usual time-dependent exponential contribution, and a reminder term that is jointly polynomially small with respect to the time and the semiclassical parameter. We also compute explicitly the main contribution of the remainder.
\end{abstract}

Keywords: Resonances; Born-Oppenheimer approximation; eigenvalue crossing; quantum evolution; survival probability.

Subject classifications: 35P15; 35C20; 35S99; 47A75.

\footnotetext{
${ }^{1}$ Aix-Marseille Université, CNRS, CPT UMR 7332, 13288 Marseille, France, and Université de Toulon, CNRS, CPT UMR 7332, $83957 \mathrm{La}$ Garde, France, briet@univ-tln.fr

${ }^{2}$ Università di Bologna, Dipartimento di Matematica, Piazza di Porta San Donato, 40127 Bologna, Italy, andre.martinez@unibo.it 


\section{INTRODUCTION}

This paper concerns the study of the behaviour in time of some quantum states describing the predissociation process of a molecular systems in the Born Oppenheimer approximation. Recall that in this context, the predissociation is connected with a resonant state of the system coming from an internal conversion from an excited state towards a dissociative state when the Born-Oppenheimer parameter $h$ is small. We refer to a recent paper by the same authors $\mathrm{BrMa}$ and references therein for more details.

Here we consider the critical case where the reference energy $E=0$ corresponds to a crossing of the confining electronic energy curve and the dissociative one. We suppose that the system has only one such crossing point. Despite the absence of tunnelling for $E$, resonances exist [FMW1]. They are of the form $\rho(h)=\lambda(h)+O\left(h^{\frac{4}{3}}\right)$ where $\lambda(h)$ is an eigenvalue (embedded in the continuous spectrum) near 0 of the decoupled operator, and their widths satisfy $\operatorname{Im} \rho(h)=O\left(h^{\frac{5}{3}}\right)$ as $h \sim 0$ (actually, under some assumption of non degeneracy of the coupling operator, one also know that $\operatorname{Im} \rho(h)<0$ : see [FMW1, FMW2]). Therefore, an attention must be paid to the dynamics of certain states having an energy close to that of the resonance.

As in the case studied in [BrMa], the initial state $\phi$ is the normalized eigenvector associated with a simple eigenvalue $\lambda(h)$ of the decoupled operator. Then, we show that for $h$ small enough, $g$ a cut-off function supported near $\lambda(h)$, and $t \in \mathbb{R}^{+}$, the survival probability satisfies,

$$
\mathcal{A}_{\phi}=\left(e^{-i t H} g(H) \phi, \phi\right)=e^{-i t \rho(h)} b(\phi, h)+r(t, \phi, h),
$$

where $b(\phi, h)=1+\mathcal{O}\left(h^{\frac{1}{3}}\right)$ and $r(t, \phi, h)=h^{\frac{2}{3}} \mathcal{O}\left(\langle h t\rangle^{-\infty}\right.$ ) (here we use the notation $\left.\langle s\rangle:=\left(1+s^{2}\right)^{\frac{1}{2}}\right)$. We actually prove this result in a situation where the inter-level coupling is a general first-order differential operator. In the physical model the coupling operator is a vector-field (see [FMW2]), and we then expect a higher order estimate on the long time part of $\mathcal{A}_{\phi}$ i.e. $r(t, \phi, h)=h^{\frac{4}{3}} \mathcal{O}\left(\langle h t\rangle^{-\infty}\right)$. This fact will be proved in a forthcoming paper [BrMa3]. 
In contrast with previous papers on similar estimates (see, e.g., CGH, CoSo, Her, $\mathrm{Hu} 2$, JeNe]), here we also focus on the precise behaviour of the remainder term $r(t, \phi, h)$. We prove,

$$
r(t, \phi, h)=\alpha h^{\frac{2}{3}} e^{-i t \lambda(h)} F(h t)+\mathcal{O}\left(h\langle h t\rangle^{-\infty}\right),
$$

where $\alpha$ behaves like a constant, and $F$ is an explicit analytic function on $\mathbb{R}^{+}$ (depending on $g$ ) that satisfies $F(0) \neq 0, F(\lambda)=\mathcal{O}\left(\langle\lambda\rangle^{-\infty}\right.$ ) (see Theorem 2.1 for the precise statement).

In view of (1.1), it turns out that the critical time $t_{c}$, within which the contribution of the exponential part of $\mathcal{A}_{\phi}$ is preponderant with respect to the remainder term, satisfies,

$$
t_{c} \geq \frac{2}{3} \frac{|\ln (h)|}{|\operatorname{Im} \rho|} .
$$

(Recall that $\operatorname{Im} \rho(h)=\mathcal{O}\left(h^{\frac{5}{3}}\right)$.) This means that for time $t \leq t_{c}$ the strong resonance effects persist, while they disappear for larger times. (Note that for the physical model, we have $\operatorname{Im} \rho(h)=\mathcal{O}\left(h^{\frac{7}{3}}\right)$ [FMW2, and we can expect that $t_{c} \geq \frac{4}{3} \frac{|\ln (h)|}{|\operatorname{Im} \rho|}$.)

Concerning the proof, in addition to the techniques introduced in FMW1 we also use some special kinds of semiclassical function spaces that permit us to considerably facilitate the estimates on the remainder term $r(t, \phi, h)$.

Let us describe the content of the paper. In section 2 we give the assumptions and the main result. The strategy of the proof involving the distortion theory will be described in section 3 . Section 4 and 5 are devoted to obtain convenient estimates on the resolvent operators. In the section $6,7,8$ and 9 we prove estimates on the remainder term in the r.h.s of (1.1). The coefficient $b(h)$ is studied in section 9 .

\section{Assumptions AND MAIN RESUlt}

We consider the semiclassical $2 \times 2$-matrix Schrödinger operator,

$$
H=\left(\begin{array}{cc}
P_{1} & h W \\
h W^{*} & P_{2}
\end{array}\right) \quad ; \quad P_{j}=h^{2} D_{x}^{2}+V_{j}(x)
$$

where, as in [FMW1, FMW2], we assume, 
Assumption (A1) $V_{1}(x), V_{2}(x)$ are real-analytic on $\mathbb{R}$ and extend to holomorphic functions in the complex domain,

$$
\Gamma=\left\{x \in \mathbb{C} ;|\operatorname{Im} x|<\varepsilon_{0}\langle\operatorname{Re} x\rangle\right\} \quad ; \quad\langle\operatorname{Re} x\rangle:=\left(1+|\operatorname{Re} x|^{2}\right)^{\frac{1}{2}},
$$

where $\varepsilon_{0}>0$ is a constant.

Assumption (A2)For $j=1,2, V_{j}$ admits limits as $\operatorname{Re} x \rightarrow \pm \infty$ in $\Gamma$, and they satisfy,

$$
\begin{aligned}
& \lim _{\substack{x \rightarrow-\infty \\
\operatorname{Re}}} V_{1}(x)>0 ; \lim _{\substack{\operatorname{Re} x \rightarrow-\infty \\
x \in \Gamma}} V_{2}(x)>0 ; \\
& \operatorname{\operatorname {lim}_{x\rightarrow +\infty }} V_{1}(x)>0 ; \lim _{\substack{\operatorname{Re} \rightarrow+\infty \\
x \in \Gamma}} V_{2}(x)<0 .
\end{aligned}
$$

Assumption (A3) One has,

$$
V_{1}^{\prime}\left(x^{*}\right)=:-\tau_{0}<0, \quad V_{1}^{\prime}(0)=: \tau_{1}>0, \quad V_{2}^{\prime}(0)=:-\tau_{2}<0,
$$

and there exists a negative number $x^{*}<0$ such that,

- $V_{1}>0$ and $V_{2}>0$ on $\left(-\infty, x^{*}\right)$;

- $V_{1}<0<V_{2}$ on $\left(x^{*}, 0\right)$;

- $V_{2}<0<V_{1}$ on $(0,+\infty)$.

Assumption (A4) $W\left(x, h D_{x}\right)$ is a first order differential operator

$$
W\left(x, h D_{x}\right)=a_{0}(x)+i a_{1}(x) h D_{x},
$$

where $a_{0}(x)$ and $a_{1}(x)$ are analytic and bounded in $\Gamma$, and real for real $x$.

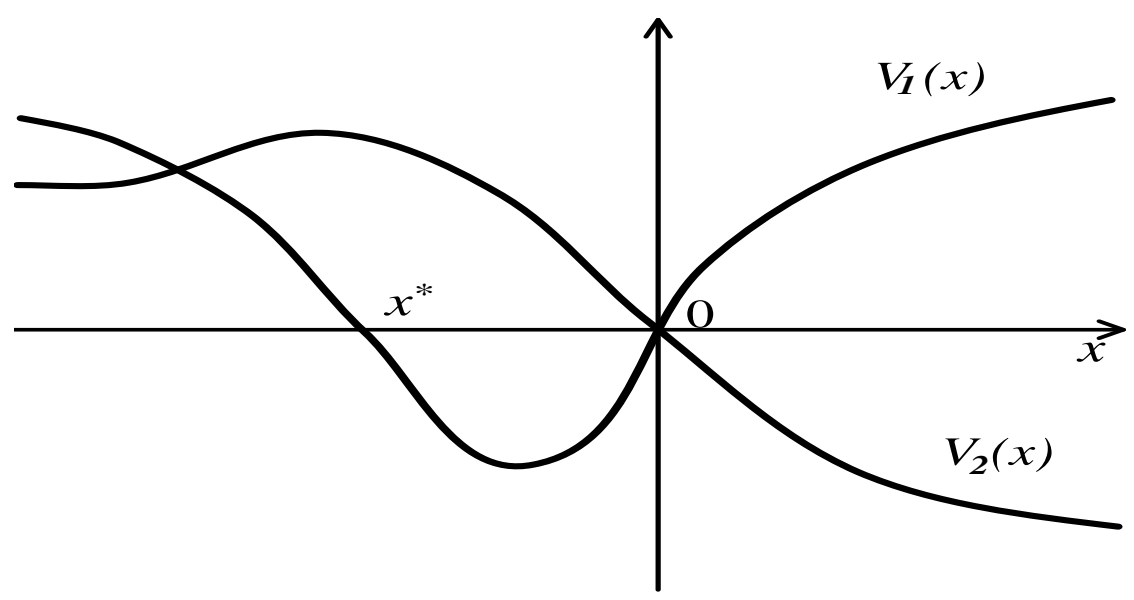

Figure 1. The two potentials 
In this situation, we know from [FMW1 that the resonances of $H$ that are inside $\mathcal{D}_{h}\left(C_{0}\right):=\left[-C_{0} h^{2 / 3}, C_{0} h^{2 / 3}\right]-i\left[0, C_{0} h\right]\left(C_{0}>0\right.$ arbitrary) are of the form,

$$
\rho_{k}(h)=e_{k}(h)+\mathcal{O}\left(h^{\frac{4}{3}}\right) \quad ; \quad \operatorname{Im} \rho_{k}(h)=\mathcal{O}\left(h^{\frac{5}{3}}\right),
$$

with $k \in \mathbb{N}$ and

$$
e_{k}(h):=\frac{-2 \mathcal{A}(0)+(2 k+1) \pi h}{2 \mathcal{A}^{\prime}(0)} ; \mathcal{A}(E):=\int_{x_{1}^{*}(E)}^{x_{1}(E)} \sqrt{E-V_{1}(t)} d t,
$$

where $x_{1}^{*}(E)$ (respectively $x_{1}(E)$ ) is the unique solution of $V_{1}(x)=E$ close to $x^{*}$ (respectively close to 0 ). In addition, at each such $e_{k}(h)$ inside $\left[-C_{0} h^{\frac{2}{3}}, C_{0} h^{\frac{2}{3}}\right]$, corresponds a unique resonance $\rho_{k}(h)$ of $H$ that satisfies (2.2). On the other hand, it is also well known (see, e.g., [HeRo]) that at each such $e_{k}(h)$ inside $\left[-C_{0} h^{\frac{2}{3}}, C_{0} h^{\frac{2}{3}}\right]$, corresponds a unique eigenvalue $E_{k}(h)$ of $P_{1}$, such that,

$$
E_{k}(h)=e_{k}(h)+\mathcal{O}\left(h^{2}\right),
$$

From now on, we fix such an eigenvalue, that is, we choose once for all an application,

$$
h \mapsto \lambda_{0}(h) \in \operatorname{Sp}\left(P_{1}\right) \cap\left[-C_{0} h^{\frac{2}{3}}, C_{0} h^{\frac{2}{3}}\right],
$$

to which corresponds a unique application,

$$
h \mapsto \rho_{0}(h) \in \operatorname{Res}(H) \cap \mathcal{D}_{h}\left(C_{0}\right),
$$

such that,

$$
\rho_{0}(h)-\lambda_{0}(h)=\mathcal{O}\left(h^{\frac{4}{3}}\right) .
$$

We also denote by $\varphi_{0}$ the real-valued normalized eigenfunction of $P_{1}$ associated with $\lambda_{0}$ (so that $W \varphi_{0}$ and $W^{*} \varphi_{0}$ are real-valued, too), and we set,

$$
\phi:=\left(\varphi_{0}, 0\right) \in L^{2}(\mathbb{R}) \oplus L^{2}(\mathbb{R}) .
$$

In particular, there exists some complex number $c_{0}=c_{0}(h) \sim 1$ such that, for $x \leq 0$,

$$
\varphi_{0}=c_{0} h^{-\frac{1}{6}} u_{1, L}^{-}\left(\lambda_{0}\right) .
$$

(Actually, by computing the $L^{2}$-norm of $u_{1, L}^{-}\left(\lambda_{0}\right)$ on $I_{L}$, one can see that $\left.c_{0}^{2}=\frac{2}{\pi} \int_{x^{*}}^{0} \frac{d x}{\sqrt{\lambda_{0}-V_{1}(x)}}+\mathcal{O}\left(h^{\frac{1}{3}}\right).\right)$

We also fix some cutoff function $g_{0} \in C_{0}^{\infty}\left(\left(-\delta_{1}, \delta_{1}\right) ;[0,1]\right)$ such that $g=1$ on $\left[-\delta_{0}, \delta_{0}\right]$ with $0<\delta_{0}<\delta_{1}<\frac{\pi}{\mathcal{A}^{\prime}(0)}$, so that, if we set,

$$
g(\lambda):=g_{0}\left(\frac{\lambda-\lambda_{0}}{h}\right),
$$


then, for $h$ small enough, $\lambda_{0}$ is the only eigenvalue of $P_{1}$ contained in the support of $g$.

We are interested in the survival amplitude associated with $g(H)^{\frac{1}{2}} \phi$,

$$
\mathcal{A}_{\phi}:=\left\langle e^{-i t H} g(H) \phi, \phi\right\rangle \text {. }
$$

In order to state our result, we define,

$$
F(\lambda):=-2 i \int_{\gamma_{0}} \frac{e^{-i \lambda z} g_{0}(\operatorname{Re} z)}{z^{2}} d z,
$$

where $\gamma_{0}$ is the oriented complex path,

$$
\gamma_{0}:=\left(-\infty,-\delta_{0}\right] \cup\left\{\delta_{0} e^{i \alpha} ; \alpha \in[\pi, 2 \pi]\right\} \cup\left[\delta_{0},+\infty\right) .
$$

In particular, let us observe that $F$ is analytic, and that $F(0) \neq 0$ (indeed, one can compute $F(0)=4 i \alpha \delta_{1}^{-1}$ with $\left.\alpha \geq 1\right)$. In addition, by integration by parts, we also see that $F(\lambda)=\mathcal{O}\left(|\lambda|^{-\infty}\right)$ as $\lambda \rightarrow \pm \infty$.

In the sequel, we denote by $\mathrm{Ai}$ and $\mathrm{Bi}$ the standard Airy functions, and for any function $f=f(s)$ we set $\check{f}(s):=f(-s)$

Our main result is,

Theorem 2.1. Under assumptions (A1)-(A4), one has,

$$
\mathcal{A}_{\phi}=e^{-i t \rho_{0}} b(h)+h^{\frac{2}{3}} q_{0}(t, h)+\mathcal{O}\left(h\langle h t\rangle^{-\infty}\right)
$$

uniformly for $h>0$ small enough and $t \in \mathbb{R}$, with,

$$
\begin{gathered}
b(h)=1+\mathcal{O}\left(h^{1 / 3}\right) ; \\
q_{0}(t, h)=4 a_{0}(0)^{2} c_{0}^{2} e^{-i t \lambda_{0}}\left[A_{0}\left(\lambda_{0} h^{-\frac{2}{3}}\right)\right]^{2} F(h t),
\end{gathered}
$$

where $F$ is defined in (2.4), and $A_{0}$ is the function,

$$
A_{0}(s):=\tau_{1}^{-\frac{1}{6}} \tau_{2}^{-\frac{1}{6}}\left(\tau_{1}+\tau_{2}\right)^{-\frac{1}{3}} \check{\mathrm{Ai}}\left(\left(\frac{\tau_{1}+\tau_{2}}{\tau_{1} \tau_{2}}\right)^{\frac{2}{3}} s\right) \text {. }
$$

\section{Preliminaries}

As in [BrMa, Section 5], we have,

$$
\mathcal{A}_{\phi}=e^{-i t \rho_{0}} b(\phi, h)+r(t, \phi, h),
$$

where $b(\phi, h)$ is the residue at $\rho_{0}$ of the meromorphic function

$$
z \mapsto-\left\langle R_{\theta}(z) \phi_{\theta}, \phi_{-\theta}\right\rangle,
$$


where, $R_{\theta}(z):=U_{\theta}\left(H_{\theta}-z\right)^{-1} U_{\theta}^{-1}$ is the distorted resolvent of $H$, and $\phi_{\theta}=\left(\varphi_{0}^{\theta}, 0\right):=U_{\theta} \phi$ is the analytic distortion of $\phi$, where $U_{\theta}$ is the analytic distorsion given by,

$$
U_{\theta} \phi(x)=\phi(x+i \theta \nu(x)),
$$

with $\nu \in C^{\infty}(\mathbb{R} ; \mathbb{R}), \nu=0$ on $\left(-\infty, x_{\infty}\right]$ for some $x_{\infty}>0, \nu(x)=x$ for $x$ large enough (see [FMW1, Section 3]).

Further, $r(t, \phi, h)$ is given by,

$$
r(t, \phi, h):=\frac{1}{2 i \pi} \int_{\gamma_{-}} e^{-i t z} g(\operatorname{Re} z)\left(\left\langle R_{\theta}(z) \phi_{\theta}, \phi_{-\theta}\right\rangle-\left\langle R_{-\theta}(z) \phi_{-\theta}, \phi_{\theta}\right\rangle\right) d z,
$$

where $\gamma_{-}$is a complex contour parametrized by $\operatorname{Re} z$, that coincides with $\mathbb{R}$ away from $\{g=1\}$, is included in $\{\operatorname{Im} z<0\}$ when $\operatorname{Re} z$ is inside $\{g=1\}$, and is chosen in such a way that it stays below $\rho_{0}$ and at a distance $\sim h$ from it.

Then, setting $v=\left(v_{1}, v_{2}\right):=R_{\theta}(z) \varphi_{\theta}$, and denoting by $P_{j}^{\theta}, W_{\theta}, W_{\theta}^{*}$ the various distorted operators, we have,

$$
\left\{\begin{array}{l}
\left(P_{1}^{\theta}-z\right) v_{1}+h W_{\theta} v_{2}=\varphi_{0}^{\theta} \\
\left(P_{2}^{\theta}-z\right) v_{2}+h W_{\theta}^{*} v_{1}=0
\end{array}\right.
$$

and thus, for $z \in \gamma_{-}$,

$$
\left\{\begin{array}{l}
v_{1}=\frac{1}{\lambda_{0}-z} \varphi_{0}^{\theta}-h\left(P_{1}^{\theta}-z\right)^{-1} W_{\theta} v_{2} \\
\left(1-M_{\theta}(z)\right) v_{2}=\frac{-h}{\lambda_{0}-z}\left(P_{2}^{\theta}-z\right)^{-1} W_{\theta}^{*} \varphi_{0}^{\theta}
\end{array}\right.
$$

with,

$$
M_{\theta}(z):=h^{2}\left(P_{2}^{\theta}-z\right)^{-1} W_{\theta}^{*}\left(P_{1}^{\theta}-z\right)^{-1} W_{\theta} .
$$

In the next sections, we will prove that, for $h$ small enough, we have $\left\|M_{\theta}(z)\right\|<$ 1 (see (5.3)). Assuming for a while this result, we conclude from (3.3) that we have,

$$
\left\{\begin{array}{l}
v_{1}=\frac{1}{\lambda_{0}-z} \varphi_{0}^{\theta}+\frac{h^{2}}{\lambda_{0}-z} \sum_{\ell \geq 0}\left(P_{1}^{\theta}-z\right)^{-1} W_{\theta} M_{\theta}(z)^{\ell}\left(P_{2}^{\theta}-z\right)^{-1} W_{\theta}^{*} \varphi_{0}^{\theta} \\
\left(1-M_{\theta}(z)\right) v_{2}=\sum_{\ell \geq 0} \frac{-h}{\lambda_{0}-z} M_{\theta}(z)^{\ell}\left(P_{2}^{\theta}-z\right)^{-1} W_{\theta}^{*} \varphi_{0}^{\theta},
\end{array}\right.
$$


As a consequence, since $\left\langle R_{\theta}(z) \phi_{\theta}, \phi_{-\theta}\right\rangle=\left\langle v_{1}, \varphi_{0}^{-\theta}\right\rangle$, and $\left\langle\varphi_{0}^{\theta}, \varphi_{0}^{-\theta}\right\rangle=\left\|\varphi_{0}\right\|^{2}=$ 1, we obtain,

$$
\begin{aligned}
& \left\langle R_{\theta}(z) \phi_{\theta}, \phi_{-\theta}\right\rangle \\
& =\frac{1}{\lambda_{0}-z}+\frac{h^{2}}{\left(\lambda_{0}-z\right)^{2}} \sum_{\ell \geq 0}\left\langle M_{\theta}(z)^{\ell}\left(P_{2}^{\theta}-z\right)^{-1} W_{\theta}^{*} \varphi_{0}^{\theta}, W_{-\theta}^{*} \varphi_{0}^{-\theta}\right\rangle .
\end{aligned}
$$

Inserting into 3.2 , we finally obtain,

$$
r(t, \phi, h)=\frac{h^{2}}{2 i \pi} \sum_{\ell \geq 0} \int_{\gamma_{-}} \frac{e^{-i t z} g(\operatorname{Re} z)}{\left(\lambda_{0}-z\right)^{2}} T_{\ell}(z) d z,
$$

with

$$
\begin{aligned}
T_{\ell}(z):=\left\langle M_{\theta}(z)^{\ell}\left(P_{2}^{\theta}-z\right)^{-1} W_{\theta}^{*} \varphi_{0}^{\theta}, W_{-\theta}^{*} \varphi_{0}^{-\theta}\right\rangle \\
-\left\langle M_{-\theta}(z)^{\ell}\left(P_{2}^{-\theta}-z\right)^{-1} W_{-\theta}^{*} \varphi_{0}^{-\theta}, W_{\theta}^{*} \varphi_{0}^{\theta}\right\rangle .
\end{aligned}
$$

Therefore,

$$
r(t, \phi, h)=r_{0}(t, \phi, h)+r_{1}(t, \phi, h)+r_{2}(t, \phi, h)
$$

with,

$$
\begin{aligned}
& r_{0}(t, \phi, h):=\frac{h^{2}}{2 i \pi} \int_{\gamma_{-}} \frac{e^{-i t z} g(\operatorname{Re} z)}{\left(\lambda_{0}-z\right)^{2}} T_{0}(z) d z \\
& r_{1}(t, \phi, h):=\frac{h^{2}}{2 i \pi} \int_{\gamma_{-}} \frac{e^{-i t z} g(\operatorname{Re} z)}{\left(\lambda_{0}-z\right)^{2}} T_{1}(z) d z \\
& r_{2}(t, \phi, h):=\frac{h^{2}}{2 i \pi} \sum_{\ell \geq 2} \int_{\gamma_{-}} \frac{e^{-i t z} g(\operatorname{Re} z)}{\left(\lambda_{0}-z\right)^{2}} T_{\ell}(z) d z,
\end{aligned}
$$

and, by an additional change of contour of integration (that brings $\gamma_{-}$onto $\mathbb{R})$, we also obtain,

$$
\begin{aligned}
& r_{0}(t, \phi, h) \\
& =\frac{h^{2}}{2 i \pi} \lim _{\varepsilon \rightarrow 0_{+}} \int_{\mathbb{R}} \frac{e^{-i t z} g(z)}{\left(\lambda_{0}+i \varepsilon-z\right)^{2}}\left\langle\left[\left(P_{2}-z-i 0\right)^{-1}-\left(P_{2}-z+i 0\right)^{-1}\right] W^{*} \varphi_{0}, W^{*} \varphi_{0}\right\rangle d z,
\end{aligned}
$$

that is, by Stone's formula,

$$
r_{0}(t, \phi, h)=h^{2}\left\langle e^{-i t P_{2}} g\left(P_{2}\right)\left(P_{2}-\lambda_{0}-i 0\right)^{-1} W^{*} \varphi_{0},\left(P_{2}-\lambda_{0}+i 0\right)^{-1} W^{*} \varphi_{0}\right\rangle .
$$

The next sections are devoted to the estimates on $\left\|M_{ \pm \theta}(z)\right\|$ and on $r_{0}(t, \phi, h)$, $r_{1}(t, \phi, h)$ and $r_{2}(t, \phi, h)$.

\section{Fundamental SOlutions}

For $z \in \mathcal{D}_{h}\left(C_{0}\right)$ and $j=1,2$, let $u_{j, L}^{ \pm}(z)=u_{j, L}^{ \pm}(z, x)$ be the global WKB solutions to $\left(P_{j}-z\right) u=0$ on $I_{L}:=(-\infty, 0]$ given, e.g., in [FMW1] (in 
particular, $u_{j, L}^{-}(z)$ decays exponentially in $x$ at $-\infty$, while $u_{j, L}^{+}(z)$ grows exponentially). Let also $u_{j, R}^{ \pm}(z)=u_{j, R}^{ \pm}(z, x)$ be the global WKB solutions to $\left(P_{j}-z\right) u=0$ on a complex neighborhood of $I_{R}:=[0,+\infty)$, such that $u_{j, R}^{-}(z)$ decays exponentially in $x$ at infinity on $I_{R}^{\theta}:=\{x+i \theta \nu(x) ; x \geq 0\}$ $(\theta>0$ fixed small enough).

In particular, we see in that $u_{2, R}^{+}(z)$ decays exponentially in $x$ at infinity on $I_{R}^{-\theta}=\overline{I_{R}^{\theta}}$.

We set,

$$
\begin{aligned}
K_{j, L}(z)[v](x):= & \frac{u_{j, L}^{+}(z, x)}{h^{2} \mathcal{W}\left[u_{j, L}^{+}(z), u_{j, L}^{-}(z)\right]} \int_{-\infty}^{x} u_{j, L}^{-}(z, t) v(t) d t \\
& +\frac{u_{j, L}^{-}(z, x)}{h^{2} \mathcal{W}\left[u_{j, L}^{+}(z), u_{j, L}^{-}(z)\right]} \int_{x}^{0} u_{j, L}^{+}(z, t) v(t) d t
\end{aligned}
$$

where $v$ is in the space $C_{b}^{0}\left(I_{L}\right)$ of bounded continuous functions on $I_{L}$;

$$
\begin{aligned}
K_{j, R}^{+}(z)[v](x):= & \frac{u_{j, R}^{-}(z, x)}{h^{2} \mathcal{W}\left[u_{j, R}^{-}(z), u_{j, R}^{+}(z)\right]} \int_{0}^{x} u_{j, R}^{+}(z, t) v(t) d t \\
& +\frac{u_{j, R}^{+}(z, x)}{h^{2} \mathcal{W}\left[u_{j, R}^{-}(z), u_{j, R}^{+}(z)\right]} \int_{x}^{+\infty} u_{j, R}^{-}(z, t) v(t) d t
\end{aligned}
$$

where $v$ is in the space $C_{b}^{0}\left(I_{R}^{+}\right)$of bounded continuous functions on $I_{R}^{+}:=I_{R}^{\theta}$, and the integrals run over $I_{R}^{+}$(see [FMW1, Section 3.2]);

$$
\begin{aligned}
K_{j, R}^{-}(z)[v](x):= & \frac{u_{j, R}^{+}(z, x)}{h^{2} \mathcal{W}\left[u_{j, R}^{+}(z), u_{j, R}^{-}(z)\right]} \int_{0}^{x} u_{j, R}^{-}(z, t) v(t) d t \\
& +\frac{u_{j, R}^{-}(z, x)}{h^{2} \mathcal{W}\left[u_{j, R}^{+}(z), u_{j, R}^{-}(z)\right]} \int_{x}^{+\infty} u_{j, R}^{+}(z, t) v(t) d t,
\end{aligned}
$$

where $v$ is in the space $C_{b}^{0}\left(I_{R}^{-}\right)$of bounded continuous functions on $I_{R}^{-}:=$ $I_{R}^{-\theta}$, and the integrals run over $I_{R}^{-}$.

Then, as in [FMW1, Section 3], we see that we have,

$$
\begin{array}{ll}
\left(P_{j}-z\right) K_{j, L}(z)=1 & \text { on } C_{b}^{0}\left(I_{L}\right) ; \\
\left(P_{j}-z\right) K_{j, R}^{ \pm}(z)=1 & \text { on } C_{b}^{0}\left(I_{R}^{ \pm}\right) .
\end{array}
$$

In the sequel, we will need the following result: 


\section{Proposition 4.1.}

$$
\begin{gathered}
\left\|K_{2, L}\right\|_{\mathcal{L}\left(L^{2}\left(I_{L}\right)\right)}+\left\|K_{1, R}^{ \pm}\right\|_{\mathcal{L}\left(L^{2}\left(I_{R}^{ \pm}\right)\right)}=\mathcal{O}\left(h^{-\frac{2}{3}}\right) \\
\left\|K_{1, L}\right\|_{\mathcal{L}\left(L^{2}\left(I_{L}\right)\right)}+\left\|K_{2, R}^{ \pm}\right\|_{\mathcal{L}\left(L^{2}\left(I_{R}^{ \pm}\right)\right)}=\mathcal{O}\left(h^{-\frac{7}{6}}\right)
\end{gathered}
$$

Proof. The proofs on $I_{L}$ and on $I_{R}^{ \pm}$are very similar, so we just give the one on $I_{L}$. Since $\mathcal{W}\left[u_{j, L}^{+}(z), u_{j, L}^{-}(z)\right] \sim h^{-\frac{2}{3}}(j=1,2)$, by 4.1) and the Schur Lemma (see, e,g, [Ma2]), it is enough to estimate,

$$
h^{-\frac{4}{3}} \sup _{x \in I_{L}} \int_{j_{L}}\left|U_{j, L}(t, x)\right| d t
$$

with

$$
U_{j, L}(t, x):=u_{j, L}^{+}(x) u_{j, L}^{-}(t) \mathbf{1}_{t \leq x}+u_{j, L}^{-}(x) u_{j, L}^{+}(t) \mathbf{1}_{x \leq t} .
$$

When $x \leq x^{*}-\delta$ with $\delta>0$ fixed arbitrarily small, we know (see, e.g., [FMW1] ) that $U_{1, L}(t, x)=\mathcal{O}\left(h^{\frac{1}{3}} e^{-c|x-t| / h}\right)$ for some constant $c>0$. Hence,

$$
h^{-\frac{4}{3}} \sup _{x \leq x^{*}-\delta} \int_{I_{L}}\left|U_{1, L}(t, x)\right| d t=\mathcal{O}(1) .
$$

When $x^{*}-\delta \leq x \leq 0$, then $\int_{-\infty}^{x^{*}-2 \delta}\left|U_{1, L}(t, x)\right| d t$ is exponentially small, while, for $t \in\left[x^{*}-2 \delta, 0\right]$, we have,

$$
U_{1, L}(t, x)=\mathcal{O}\left(h^{\frac{1}{6}}|t|^{-\frac{1}{4}}\left|t-x^{*}\right|^{-\frac{1}{4}}\right) .
$$

We deduce,

$$
h^{-\frac{4}{3}} \sup _{x^{*}-\delta \leq x \leq 0} \int_{I_{L}}\left|U_{1, L}(t, x)\right| d t=\mathcal{O}\left(h^{-\frac{7}{6}}\right),
$$

and thus,

$$
\left\|K_{1, L}\right\|_{\mathcal{L}\left(L^{2}\left(I_{L}\right)\right)}=\mathcal{O}\left(h^{-\frac{7}{6}}\right) .
$$

Concerning $K_{2, L}$, the same estimate $U_{2, L}(t, x)=\mathcal{O}\left(h^{\frac{1}{3}} e^{-c|x-t| / h}\right)$ is valid for $x \leq-\delta(\delta>0$ arbitrarily small, $c=c(\delta)>0)$. Therefore,

$$
h^{-\frac{4}{3}} \sup _{x \leq-\delta} \int_{I_{L}}\left|U_{2, L}(t, x)\right| d t=\mathcal{O}(1) .
$$

Now, if $x \in\left[-\delta,-C h^{\frac{2}{3}}\right]$ (with a constant $C>0$ sufficiently large), we can write,

$$
\begin{aligned}
h^{-\frac{4}{3}} \int_{I_{L}}\left|U_{2, L}(t, x)\right| d t= & h^{-\frac{4}{3}} \int_{-\infty}^{-2 \delta}\left|U_{2, L}(t, x)\right| d t+h^{-\frac{4}{3}} \int_{-2 \delta}^{-C h^{\frac{2}{3}}}\left|U_{2, L}(t, x)\right| d t \\
& +h^{-\frac{4}{3}} \int_{-C h^{\frac{2}{3}}}^{0}\left|U_{2, L}(t, x)\right| d t
\end{aligned}
$$


where the first term of the right hand side is exponentially small, while the last term is $\mathcal{O}\left(h^{-\frac{2}{3}}\right)$. The middle term can be estimated by,

$$
h^{-\frac{4}{3}} \int_{-2 \delta}^{-C h^{\frac{2}{3}}}\left|U_{2, L}(t, x)\right| d t=\mathcal{O}\left(h^{-\frac{4}{3}+\frac{1}{6}}\right) \int_{-2 \delta}^{-C h^{\frac{2}{3}}} \frac{e^{-\left|t^{3 / 2}-x^{3 / 2}\right| / h}}{|t|^{\frac{1}{4}}} d t,
$$

and first dividing the integral into $\int_{-2 \delta}^{x}+\int_{x}^{-C h^{\frac{2}{3}}}$, then making the change of variable $t \mapsto-(h t)^{\frac{2}{3}}$, we obtain,

$$
\begin{aligned}
h^{-\frac{4}{3}} \int_{-2 \delta}^{-C h^{\frac{2}{3}}}\left|U_{2, L}(t, x)\right| d t= & \mathcal{O}\left(h^{-\frac{4}{3}+\frac{1}{6}+\frac{2}{3}-\frac{1}{6}}\right) e^{-|x|^{\frac{3}{2}} / h} \int_{C^{\frac{3}{2}}}^{|x|^{\frac{3}{2}} / h} \frac{e^{t}}{\sqrt{t}} d t \\
& +\mathcal{O}\left(h^{-\frac{2}{3}}\right) e^{|x|^{\frac{3}{2}} / h} \int_{|x|^{\frac{3}{2}} / h}^{(2 \delta)^{\frac{3}{2}} / h} \frac{e^{-t}}{\sqrt{t}} d t
\end{aligned}
$$

and thus,

$$
h^{-\frac{4}{3}} \int_{-2 \delta}^{-C h^{\frac{2}{3}}}\left|U_{2, L}(t, x)\right| d t=\mathcal{O}\left(h^{-\frac{2}{3}}\right) .
$$

Finally, if $x \in\left[-C h^{\frac{2}{3}}, 0\right]$, the same argument (but this time without dividing the integral $\left.\int_{-2 \delta}^{-C h^{\frac{2}{3}}}\right)$ directly gives $h^{-\frac{4}{3}} \int_{-2 \delta}^{-C h^{\frac{2}{3}}}\left|U_{2, L}(t, x)\right| d t=\mathcal{O}\left(h^{-\frac{2}{3}}\right)$, and the estimate on $\left\|K_{2, L}\right\|_{\mathcal{L}\left(L^{2}\left(I_{L}\right)\right)}$ follows. Similar arguments (but with $x^{*}$ substituted by some large enough value of $x$ ) also apply on $I_{R}^{ \pm}$, and complete the proof of the proposition.

\section{Resolvents}

We consider the space $\mathcal{S}$ of functions $\varphi \in C^{\infty}(\mathbb{R})$ that are analytic on $\left[x_{\infty},+\infty\right)$ and admit a holomorphic extension (still denoted by $\varphi$ ) near $\Gamma_{\delta}:=\left\{x \in \mathbb{C} ; \operatorname{Re} x \geq x_{\infty},|\operatorname{Im} x| \leq \delta \operatorname{Re} x\right\}$ for some $\delta>0$, and that are exponentially small at infinity both on $\mathbb{R}_{-}$and on $\Gamma_{\delta}$.

In particular, for all $\varphi \in \mathcal{S}$, we have $K_{1, R}^{+}(z)[\varphi]=K_{1, R}^{-}(z)[\varphi]=: K_{1, R}(z)[\varphi]$ on $\mathbb{R}_{+} \cup \Gamma_{\delta}$.

For $z \in \mathcal{D}_{h}\left(C_{0}\right) \cap\{ \pm \operatorname{Im} z>0\}$ and $j=1,2$, we denote by $R_{j}^{ \pm}(z)=\left(P_{j}-z\right)^{-1}$ the resolvent of $P_{j}$ in $z$, referred to as the incoming (respectively out-going) resolvent of $P_{j}$ in $z$.

Then, for $\varphi \in \mathcal{S}$, the next Proposition will show that $R_{j}^{ \pm}(z) \varphi$ extend analytically to $z \in \mathcal{D}_{h}\left(C_{0}\right)\left(z \notin \operatorname{Sp}\left(P_{1}\right)\right.$ in the case $\left.j=1\right)$, and we use the same notations for their extensions. Obviously, in the case $j=1$, one also has $R_{1}^{+}(z) \varphi=R_{1}^{-}(z) \varphi$ for $z \in \mathcal{D}_{h}\left(C_{0}\right) \backslash \operatorname{Sp}\left(P_{1}\right)$. 
Finally, for $\varphi \in \mathcal{S}$, we denote by $\varphi_{L}$ its restriction to $I_{L}$ and by $\varphi_{R}$ its restriction to $\mathbb{R}_{+} \cup \Gamma_{\delta}$.

Proposition 5.1. (i) For all $\varphi \in \mathcal{S}, z \in \mathcal{D}_{h}\left(C_{0}\right) \backslash \operatorname{Sp}\left(P_{1}\right)$, and $x \leq 0$, one has,

$$
R_{1}(z) \varphi(x)=K_{1, L}(z)\left[\varphi_{L}\right](x)+\alpha_{L}(z)[\varphi] u_{1, L}^{-}(z, x),
$$

with,

$$
\alpha_{L}(z)[\varphi]=\alpha_{L, L}(z)\left[\varphi_{L}\right]+\alpha_{L, R}(z)\left[\varphi_{R}\right]
$$

$\alpha_{L, L}(z)\left[\varphi_{L}\right]:=\frac{h^{-2} \mathcal{W}\left(u_{1, L}^{+}(z), u_{1, R}^{-}(z)\right)}{\mathcal{W}\left(u_{1, R}^{-}(z), u_{1, L}^{-}(z)\right) \mathcal{W}\left(u_{1, L}^{+}(z), u_{1, L}^{-}(z)\right)} \int_{-\infty}^{0} u_{1, L}^{-}(z, t) \varphi_{L}(t) d t$

$\alpha_{L, R}(z)\left[\varphi_{R}\right]:=\frac{h^{-2}}{\mathcal{W}\left(u_{1, R}^{-}(z), u_{1, L}^{-}(z)\right)} \int_{0}^{+\infty} u_{1, R}^{-}(z, t) \varphi_{R}(t) d t$.

(ii) For all $\varphi \in \mathcal{S}, z \in \mathcal{D}_{h}\left(C_{0}\right) \backslash \operatorname{Sp}\left(P_{1}\right)$, and $x \in \mathbb{R}_{+} \cup \Gamma_{\delta}$, one has,

$$
R_{1}(z) \varphi(x)=K_{1, R}(z)\left[\varphi_{R}\right](x)+\alpha_{R}(z)[\varphi] u_{1, R}^{-}(z, x),
$$

with,

$$
\alpha_{R}(z)[\varphi]=\alpha_{R, L}(z)\left[\varphi_{L}\right]+\alpha_{R, R}(z)\left[\varphi_{R}\right]
$$

$$
\begin{aligned}
\alpha_{R, L}(z)\left[\varphi_{L}\right] & :=\frac{h^{-2}}{\mathcal{W}\left(u_{1, R}^{-}(z), u_{1, L}^{-}(z)\right)} \int_{-\infty}^{0} u_{1, L}^{-}(z, t) \varphi_{L}(t) d t \\
\alpha_{R, R}(z)\left[\varphi_{R}\right] & :=\frac{h^{-2} \mathcal{W}\left(u_{1, L}^{-}(z), u_{1, R}^{+}(z)\right)}{\mathcal{W}\left(u_{1, R}^{-}(z), u_{1, L}^{-}(z)\right) \mathcal{W}\left(u_{1, R}^{-}(z), u_{1, R}^{+}(z)\right)} \int_{0}^{+\infty} u_{1, R}^{-}(z, t) \varphi_{R}(t) d t .
\end{aligned}
$$

(iii) For all $\varphi \in \mathcal{S}, z \in \mathcal{D}_{h}\left(C_{0}\right)$, and $x \leq 0$, one has,

$$
R_{2}^{ \pm}(z) \varphi(x)=K_{2, L}(z)\left[\varphi_{L}\right](x)+\beta_{L}^{ \pm}(z)[\varphi] u_{2, L}^{-}(z, x),
$$

with,

$$
\beta_{L}^{ \pm}(z)[\varphi]=\beta_{L, L}^{ \pm}(z)\left[\varphi_{L}\right]+\beta_{L, R}^{ \pm}(z)\left[\varphi_{R}\right]
$$

$\beta_{L, L}^{ \pm}(z)\left[\varphi_{L}\right]:=\frac{h^{-2} \mathcal{W}\left(u_{2, L}^{+}(z), u_{2, R}^{\mp}(z)\right)}{\mathcal{W}\left(u_{2, R}^{\mp}(z), u_{2, L}^{-}(z)\right) \mathcal{W}\left(u_{2, L}^{+}(z), u_{2, L}^{-}(z)\right)} \int_{-\infty}^{0} u_{2, L}^{-}(z, t) \varphi_{L}(t) d t$ $\beta_{L, R}^{ \pm}(z)\left[\varphi_{R}\right]:=\frac{h^{-2}}{\mathcal{W}\left(u_{2, R}^{\mp}(z), u_{2, L}^{-}(z)\right)} \int_{I_{R}^{ \pm}} u_{2, R}^{\mp}(z, t) \varphi_{R}(t) d t$.

(iv) For all $\varphi \in \mathcal{S}, z \in \mathcal{D}_{h}\left(C_{0}\right)$, and $x \in I_{R}^{ \pm}$, one has,

$$
R_{2}^{ \pm}(z) \varphi(x)=K_{2, R}^{ \pm}(z)\left[\varphi_{R}\right](x)+\beta_{R}^{ \pm}(z)[\varphi] u_{2, R}^{\mp}(z, x),
$$


with,

$$
\begin{gathered}
\beta_{R}^{ \pm}(z)[\varphi]=\beta_{R, L}^{ \pm}(z)\left[\varphi_{L}\right]+\beta_{R, R}^{ \pm}(z)\left[\varphi_{R}\right] \\
\beta_{R, L}^{ \pm}(z)\left[\varphi_{L}\right]:=\frac{h^{-2}}{\mathcal{W}\left(u_{2, R}^{\mp}(z), u_{2, L}^{-}(z)\right)} \int_{-\infty}^{0} u_{2, L}^{-}(z, t) \varphi_{L}(t) d t \\
\beta_{R, R}^{ \pm}(z)\left[\varphi_{R}\right]:=\frac{h^{-2} \mathcal{W}\left(u_{2, L}^{-}(z), u_{2, R}^{ \pm}(z)\right)}{\mathcal{W}\left(u_{2, R}^{\mp}(z), u_{2, L}^{-}(z)\right) \mathcal{W}\left(u_{2, R}^{\mp}(z), u_{2, R}^{ \pm}(z)\right)} \int_{I_{R}^{ \pm}} u_{2, R}^{\mp}(z, t) \varphi_{R}(t) d t .
\end{gathered}
$$

Remark 5.2. In particular, by [FMW1, Appendix], for $z \in \gamma_{-}$we have,

$$
\begin{aligned}
& \alpha_{L, L}(z)\left[\varphi_{L}\right]=\left(\frac{\pi}{4} h^{-\frac{4}{3}} \tan \frac{\mathcal{A}(z)}{h}+\mathcal{O}\left(h^{-1}\right)\right) \int_{-\infty}^{0} u_{1, L}^{-}(z, t) \varphi_{L}(t) d t ; \\
& \alpha_{L, R}(z)\left[\varphi_{R}\right]=\left(\frac{\pi}{4} h^{-\frac{4}{3}}\left(\cos \frac{\mathcal{A}(z)}{h}\right)^{-1}+\mathcal{O}\left(h^{-1}\right)\right) \int_{0}^{+\infty} u_{1, R}^{-}(z, t) \varphi_{R}(t) d t ; \\
& \alpha_{R, L}(z)\left[\varphi_{L}\right]=\left(\frac{\pi}{4} h^{-\frac{4}{3}}\left(\cos \frac{\mathcal{A}(z)}{h}\right)^{-1}+\mathcal{O}\left(h^{-1}\right)\right) \int_{-\infty}^{0} u_{1, L}^{-}(z, t) \varphi_{L}(t) d t ; \\
& \alpha_{R, R}(z)\left[\varphi_{L}\right]=\left(\frac{\pi}{4} h^{-\frac{4}{3}} \tan \frac{\mathcal{A}(z)}{h}+\mathcal{O}\left(h^{-1}\right)\right) \int_{0}^{+\infty} u_{1, R}^{-}(z, t) \varphi_{R}(t) d t ; \\
& \beta_{L, L}^{ \pm}(z)\left[\varphi_{L}\right]=\left( \pm i \frac{\pi}{4} h^{-\frac{4}{3}}+\mathcal{O}\left(h^{-1}\right)\right) \int_{-\infty}^{0} u_{2, L}^{-}(z, t) \varphi_{L}(t) d t ; \\
& \beta_{L, R}^{+}(z)\left[\varphi_{R}\right]=\left(\frac{\pi}{\sqrt{2}} e^{i \frac{\pi}{4}} h^{-\frac{4}{3}}+\mathcal{O}\left(h^{-1}\right)\right) \int_{I_{R}^{+}} u_{2, R}^{-}(z, t) \varphi_{R}(t) d t ; \\
& \beta_{L, R}^{-}(z)\left[\varphi_{R}\right]=-\left(\frac{\pi}{2 \sqrt{2}} e^{i \frac{\pi}{4}} h^{-\frac{4}{3}}+\mathcal{O}\left(h^{-1}\right)\right) \int_{I_{R}^{-}} u_{2, R}^{+}(z, t) \varphi_{R}(t) d t ; \\
& \beta_{R, L}^{+}(z)\left[\varphi_{L}\right]=\left(\frac{\pi}{\sqrt{2}} e^{i \frac{\pi}{4}} h^{-\frac{4}{3}}+\mathcal{O}\left(h^{-1}\right)\right) \int_{-\infty}^{0} u_{2, L}^{-}(z, t) \varphi_{L}(t) d t ; \\
& \beta_{R, L}^{-}(z)\left[\varphi_{L}\right]=-\left(\frac{\pi}{2 \sqrt{2}} e^{i \frac{\pi}{4}} h^{-\frac{4}{3}}+\mathcal{O}\left(h^{-1}\right)\right) \int_{-\infty}^{0} u_{2, L}^{-}(z, t) \varphi_{L}(t) d t ; \\
& \beta_{R, R}^{+}(z)\left[\varphi_{R}\right]=\left(\pi h^{-\frac{4}{3}}+\mathcal{O}\left(h^{-1}\right)\right) \int_{I_{R}^{+}} u_{2, R}^{-}(z, t) \varphi_{R}(t) d t ; \\
& \beta_{R, R}^{-}(z)\left[\varphi_{R}\right]=-\left(\frac{\pi}{4} h^{-\frac{4}{3}}+\mathcal{O}\left(h^{-1}\right)\right) \int_{I_{R}^{-}} u_{2, R}^{+}(z, t) \varphi_{R}(t) d t,
\end{aligned}
$$

with,

$$
\mathcal{A}(z):=\int_{x_{1}^{*}(z)}^{x_{1}(z)} \sqrt{z-V_{1}(t)} d t
$$

where $x_{1}^{*}(z)$ (respectively $x_{1}(z)$ ) is the unique solution of $V_{1}(x)=z$ close to $x^{*}$ (respectively close to 0 ).

Proof. We only prove (i)-(ii), since (iii)-(iv) follow along the same lines. We set,

$$
\psi:=R_{1}(z) \varphi \quad ; \quad \psi_{1, L}:=K_{1, L}(z) \varphi \quad ; \quad \psi_{1, R}:=K_{1, R}(z) \varphi
$$


Then by construction we have,

$$
\begin{aligned}
& \left(P_{1}-z\right)\left(\psi-\psi_{1, L}\right)=\left(P_{1}-z\right)\left(\psi-\psi_{1, R}\right)=0 ; \\
& \psi-\psi_{1, L} \in L^{2}\left(I_{L}\right) \quad ; \quad \psi-\psi_{1, R} \in L^{2}\left(I_{R}^{ \pm}\right) .
\end{aligned}
$$

Therefore, there exist two complex numbers $\alpha_{L}=\alpha_{L}(z)$ and $\alpha_{R}=\alpha_{R}(z)$ such that,

$$
\psi-\psi_{1, L}=\alpha_{L} u_{1, L}^{-} \quad ; \quad \psi-\psi_{1, R}=\alpha_{R} u_{1, R}^{-} .
$$

In order to compute $\alpha_{L}$ and $\alpha_{R}$, we write that $\psi$ must be $C^{1}$ at 0 . We find the system,

$$
\left\{\begin{array}{l}
\alpha_{L} u_{1, L}^{-}(0)-\alpha_{R} u_{1, R}^{-}(0)=\psi_{1, R}(0)-\psi_{1, L}(0) ; \\
\alpha_{L}\left[u_{1, L}^{-}\right]^{\prime}(0)-\alpha_{R}\left[u_{1, R}^{-}\right]^{\prime}(0)=\psi_{1, R}^{\prime}(0)-\psi_{1, L}^{\prime}(0),
\end{array}\right.
$$

and, using that,

$$
\begin{aligned}
& \psi_{1, L}(0)=\frac{u_{1 . L}^{+}(0)}{h^{2} \mathcal{W}\left(u_{1, L}^{+}, u_{1, L}^{-}\right)} \int_{-\infty}^{0} u_{1, L}^{-}(t) \varphi(t) d t ; \\
& \psi_{1, L}^{\prime}(0)=\frac{\left[u_{1 . L}^{+}\right]^{\prime}(0)}{h^{2} \mathcal{W}\left(u_{1, L}^{+}, u_{1, L}^{-}\right)} \int_{-\infty}^{0} u_{1, L}^{-}(t) \varphi(t) d t ; \\
& \psi_{1, R}(0)=\frac{u_{1 . R}^{+}(0)}{h^{2} \mathcal{W}\left(u_{1, R}^{-}, u_{1, R}^{+}\right)} \int_{0}^{+\infty} u_{1, R}^{-}(t) \varphi(t) d t ; \\
& \psi_{1, R}^{\prime}(0)=\frac{\left[u_{1 . R}^{+}\right]^{\prime}(0)}{h^{2} \mathcal{W}\left(u_{1, R}^{-}, u_{1, R}^{+}\right)} \int_{0}^{+\infty} u_{1, R}^{-}(t) \varphi(t) d t,
\end{aligned}
$$

the result follows by straightforward computations.

As a consequence of the previous proposition, we have,

Corollary 5.3. For $z \in \gamma_{-}$, one has,

$$
\begin{gathered}
\left\|R_{2}^{ \pm}(z)\right\|_{\mathcal{L}\left(L^{2}\left(I_{L} \cup I_{R}^{ \pm}\right)\right)}=\mathcal{O}\left(h^{-1-1 / 6}\right) ; \\
\left\|h^{2} R_{2}^{ \pm}(z) W^{*} R_{1}(z) W\right\|_{\mathcal{L}\left(L^{2}\left(I_{L} \cup I_{R}^{ \pm}\right)\right)}=\mathcal{O}\left(h^{1 / 6}\right) .
\end{gathered}
$$

Remark 5.4. In particular, the operators $M_{ \pm \theta}$ introduced in (3.4) also satisfy,

$$
\left\|M_{ \pm \theta}(z)\right\|_{\mathcal{L}\left(L^{2}(\mathbb{R})\right)}=\mathcal{O}\left(h^{1 / 6}\right)
$$

Proof. We first observe that, by construction (see, e.g., [FMW1]), we have,

$$
\left\|u_{2, L}^{-}\right\|_{L^{2}\left(I_{L}\right)}=\mathcal{O}\left(h^{\frac{1}{3}}\right) \quad ; \quad\left\|u_{2, R}^{\mp}\right\|_{L^{2}\left(I_{R}^{ \pm}\right)}=\mathcal{O}\left(h^{\frac{1}{6}}\right) .
$$


Using Remark 5.2, we deduce that, for $z \in \gamma_{-}$and $S=L, R$, we have,

Therefore,

$$
\begin{aligned}
\left|\beta_{S, L}^{ \pm}(z)\left[\varphi_{L}\right]\right| & =\mathcal{O}\left(h^{-1}\right)\left\|\varphi_{L}\right\|_{L^{2}\left(I_{L}\right)} \\
\left|\beta_{S, R}^{ \pm}(z)\left[\varphi_{R}\right]\right| & =\mathcal{O}\left(h^{-\frac{7}{6}}\right)\left\|\varphi_{R}\right\|_{L^{2}\left(I_{R}^{ \pm}\right)} .
\end{aligned}
$$

$$
\left\|\beta_{L}^{ \pm}(z)[\varphi] u_{2, L}^{-}\right\|_{L^{2}\left(I_{L}\right)}+\left\|\beta_{R}^{ \pm}(z)[\varphi] u_{2, R}^{\mp}\right\|_{L^{2}\left(I_{R}^{ \pm}\right)}=\mathcal{O}\left(h^{-1}\right)\|\varphi\|_{L^{2}\left(I_{L} \cup I_{R}^{ \pm}\right)} .
$$

Moreover, by Proposition 4.1, we also have,

$$
\left\|K_{2, L}(z)\left[\varphi_{L}\right]\right\|_{L^{2}\left(I_{L}\right)}+\left\|K_{2, R}^{ \pm}(z)\left[\varphi_{R}\right]\right\|_{L^{2}\left(I_{R}^{ \pm}\right)}=\mathcal{O}\left(h^{-\frac{7}{6}}\right)\left\|\varphi_{R}\right\|_{L^{2}\left(I_{R}^{ \pm}\right)} .
$$

Then, 5.1) follows from Proposition 5.1 (iii)-(iv).

Concerning (5.2), in order to simplify the notations we write the detailed proof with $W=W^{*}=\mathbf{1}$, and then we explain how to deduce the result for the actual $W, W^{*}$. We set,

$$
K_{0}^{ \pm}(z):=h^{2} R_{2}^{ \pm}(z) R_{1}(z),
$$

and we first observe that, for $z \in \gamma_{-}$, we have $\left\|R_{1}(z)\right\|=\mathcal{O}\left(h^{-1}\right)$, so that, by (5.1), a mere estimate with the product of the norms gives $\left\|K_{0}^{ \pm}(z)\right\|=$ $\mathcal{O}\left(h^{-\frac{1}{6}}\right)$. The improvement into $\mathcal{O}\left(h^{\frac{1}{6}}\right)$ will actually follow from the fact that $R_{2}(z)$ is better on $I_{L}$, while $R_{1}(z)$ is better on $I_{R}^{ \pm}$.

Using Proposition 5.1 (and dropping the parameter $z$ ), we have,

- On $I_{L}$

$$
\begin{aligned}
K_{0}^{ \pm} \varphi= & h^{2} K_{2, L} K_{1, L} \varphi_{L}+h^{2} \alpha_{L}[\varphi] K_{2, L} u_{1, L}^{-}+h^{2} \beta_{L, L}^{ \pm}\left[K_{1, L} \varphi_{L}\right] u_{2, L}^{-} \\
& +h^{2} \alpha_{L}[\varphi] \beta_{L, L}^{ \pm}\left[u_{1, L}^{-}\right] u_{2, L}^{-}+h^{2} \beta_{L, R}^{ \pm}\left[K_{1, R} \varphi_{R}\right] u_{2, L}^{-} \\
& +h^{2} \alpha_{R}[\varphi] \beta_{L, R}^{ \pm}\left[u_{1, R}^{-}\right] u_{2, L}^{-}
\end{aligned}
$$

- On $I_{R}^{ \pm}$,

$$
\begin{aligned}
K_{0}^{ \pm} \varphi= & h^{2} K_{2, R} K_{1, R} \varphi_{R}+h^{2} \alpha_{R}[\varphi] K_{2, R} u_{1, R}^{-}+h^{2} \beta_{R, L}^{ \pm}\left[K_{1, L} \varphi_{L}\right] u_{2, R}^{\mp} \\
& +h^{2} \alpha_{L}[\varphi] \beta_{R, L}^{ \pm}\left[u_{1, L}^{-}\right] u_{2, R}^{\mp}+h^{2} \beta_{R, R}^{ \pm}\left[K_{1, R} \varphi_{R}\right] u_{2, R}^{\mp} \\
& +h^{2} \alpha_{R}[\varphi] \beta_{R, R}^{ \pm}\left[u_{1, R}^{-}\right] u_{2, R}^{\mp} .
\end{aligned}
$$

Since the studies on $I_{L}$ and on $I_{R}^{ \pm}$are similar, we detail the proof for $I_{L}$ only. In view of (5.5), we have six terms to examine. We first show,

Lemma 5.5. One has,

$$
\begin{aligned}
\left\|h^{2} \alpha_{L}[\varphi] \beta_{L, L}^{ \pm}\left[u_{1, L}^{-}\right] u_{2, L}^{-}\right\|_{L^{2}\left(I_{L}\right)} & =\mathcal{O}\left(h^{\frac{1}{2}}\right)\|\varphi\|_{L^{2}\left(I_{L} \cup I_{R}^{ \pm}\right)} \\
\left\|h^{2} \alpha_{R}[\varphi] \beta_{L, R}^{ \pm}\left[u_{1, R}^{-}\right] u_{2, L}^{-}\right\|_{L^{2}\left(I_{L}\right)} & =\mathcal{O}\left(h^{\frac{1}{2}}\right)\|\varphi\|_{L^{2}\left(I_{L} \cup I_{R}^{ \pm}\right)}
\end{aligned}
$$


Proof. Since $\left\|u_{1, L}^{-}\right\|_{L^{2}\left(I_{L}\right)}$ and $\left\|u_{2, R}^{\mp}\right\|_{L^{2}\left(I_{R}^{ \pm}\right)}$are of size $\sim h^{\frac{1}{6}}$, while $\left\|u_{1, R}^{-}\right\|_{L^{2}\left(I_{R}^{ \pm}\right)}$ and $\left\|u_{2, L}^{-}\right\|_{L^{2}\left(I_{L}\right)}$ are of size $\sim h^{\frac{1}{3}}$, by Cauchy-Schwarz inequality we see on Remark 5.2 that we have,

$$
\left\|h^{2} \alpha_{L}[\varphi] u_{2, L}^{-}\right\|_{L^{2}\left(I_{L}\right)}+\left\|h^{2} \alpha_{R}[\varphi] u_{2, L}^{-}\right\|_{L^{2}\left(I_{L}\right)}=\mathcal{O}\left(h^{\frac{7}{6}}\right)\|\varphi\|_{L^{2}\left(I_{L} \cup I_{R}^{ \pm}\right)},
$$

and it remains to estimate $\left|\beta_{L, L}^{ \pm}\left[u_{1, L}^{-}\right]\right|$and $\left|\beta_{L, R}^{ \pm}\left[u_{1, R}^{-}\right]\right|$. Both can be upper bounded by,

$$
C h^{-\frac{4}{3}} \int_{0}^{C h^{\frac{2}{3}}} d t+C h^{-\frac{4}{3}} \int_{C h^{\frac{2}{3}}}^{\delta} \frac{h^{\frac{1}{3}}}{\sqrt{t}} e^{-c t^{3 / 2} / h} d t+C h^{-\frac{4}{3}} \int_{\delta}^{+\infty} h^{\frac{1}{3}} e^{-c t / h},
$$

(with $C>0$ large enough constant, and $c, \delta>0$ small enough constants), and thus are $\mathcal{O}\left(h^{-\frac{2}{3}}\right)$, and the result follows.

Lemma 5.6.

$$
\left\|h^{2} \alpha_{L}[\varphi] K_{2, L} u_{1, L}^{-}\right\|_{L^{2}\left(I_{L}\right)}=\mathcal{O}\left(h^{\frac{1}{3}}\right)\|\varphi\|_{L^{2}\left(I_{L} \cup I_{R}^{ \pm}\right)} .
$$

Proof. Using again Remark 5.2, we have,

$$
\left\|h^{2} \alpha_{L}[\varphi] K_{2, L} u_{1, L}^{-}\right\|_{L^{2}\left(I_{L}\right)}=\mathcal{O}\left(h^{\frac{5}{6}}\right)\|\varphi\|_{L^{2}\left(I_{L} \cup I_{R}^{ \pm}\right)}\left\|K_{2, L} u_{1, L}^{-}\right\|_{L^{2}\left(I_{L}\right)},
$$

and it remains to estimate $\left\|K_{2, L} u_{1, L}^{-}\right\|_{L^{2}\left(I_{L}\right)}$. Applying Proposition 4.1, we obtain,

$$
\left\|K_{2, L} u_{1, L}^{-}\right\|_{L^{2}\left(I_{L}\right)}=\mathcal{O}\left(h^{-\frac{2}{3}}\right)\left\|u_{1, L}^{-}\right\|_{L^{2}\left(I_{L}\right)}=\mathcal{O}\left(h^{-\frac{1}{2}}\right),
$$

and the result follows.

\section{Lemma 5.7.}

$$
\begin{aligned}
\left\|h^{2} \beta_{L, L}^{ \pm}\left[K_{1, L} \varphi_{L}\right] u_{2, L}^{-}\right\|_{L^{2}\left(I_{L}\right)}+\| h^{2} \beta_{L, R}^{ \pm}\left[K_{1, R} \varphi_{R}\right] & u_{2, L}^{-} \|_{L^{2}\left(I_{L}\right)} \\
& =\mathcal{O}\left(h^{\frac{1}{6}}\right)\|\varphi\|_{L^{2}\left(I_{L} \cup I_{R}^{ \pm}\right)}
\end{aligned}
$$

Proof. Since $\left\|u_{2, L}^{-}\right\|_{L^{2}\left(I_{L}\right)}=\mathcal{O}\left(h^{\frac{1}{3}}\right)$, it is enough to prove that $\beta_{L, L}^{ \pm}\left[K_{1, L} \varphi_{L}\right]$ and $\beta_{L, R}^{ \pm}\left[K_{1, R} \varphi_{R}\right]$ are $\mathcal{O}\left(h^{-\frac{13}{6}}\right)\|\varphi\|_{L^{2}\left(I_{L} \cup I_{R}^{ \pm}\right)}$. By Remark 5.2 and Proposition 4.1, we have,

$$
\begin{aligned}
\beta_{L, L}^{ \pm}\left[K_{1, L} \varphi_{L}\right] & =\mathcal{O}\left(h^{-1}\right)\left\|K_{1, L} \varphi_{L}\right\|_{L^{2}\left(I_{L}\right)}=\mathcal{O}\left(h^{-1-\frac{7}{6}}\right)\left\|\varphi_{L}\right\|_{L^{2}\left(I_{L}\right)}, \\
\beta_{L, R}^{ \pm}\left[K_{1, R} \varphi_{R}\right] & =\mathcal{O}\left(h^{-\frac{7}{6}}\right)\left\|K_{1, R} \varphi_{R}\right\|_{L^{2}\left(I_{R}^{ \pm}\right)}=\mathcal{O}\left(h^{-\frac{7}{6}-\frac{2}{3}}\right)\left\|\varphi_{R}\right\|_{L^{2}\left(I_{R}^{ \pm}\right)},
\end{aligned}
$$

and the result follows.

\section{Lemma 5.8.}

$$
\left\|h^{2} K_{2, L} K_{1, L}\right\|_{\mathcal{L}\left(L^{2}\left(I_{L}\right)\right)}=\mathcal{O}\left(h^{\frac{1}{6}}\right)
$$


Proof. It is an immediate consequence of Proposition 4.1.

Using (5.5), we conclude from Lemmas 5.55 .8 that we have,

$$
\left\|K_{0}^{ \pm} \varphi\right\|_{L^{2}\left(I_{L}\right)}=\mathcal{O}\left(h^{\frac{1}{6}}\right)\|\varphi\|_{L^{2}\left(I_{L} \cup I_{R}^{ \pm}\right)} .
$$

Analogous arguments lead to the same estimate on $I_{R}^{ \pm}$, and thus, we have proved,

$$
\left\|K_{0}^{ \pm}\right\|_{\mathcal{L}\left(L^{2}\left(I_{L} \cup I_{R}^{ \pm}\right)\right)}=\mathcal{O}\left(h^{\frac{1}{6}}\right) .
$$

Concerning the result for $\left\|h^{2} R_{2}^{ \pm}(z) W^{*} R_{1}(z) W\right\|$, we first observe that the previous proof works without changes for $\left\|h^{2} R_{2}^{ \pm}(z) f R_{1}(z) g\right\|$ if $f, g$ are bounded multiplication operators, and also for $\left\|h^{2} R_{2}^{ \pm}(z) f h D_{x} R_{1}(z) g\right\|$ because the estimates on $h\left[u_{1, L}^{ \pm}\right]^{\prime}$ and $h\left[u_{1, R}^{ \pm}\right]^{\prime}$ are the same as (and at some places even better than) those on $u_{1, L}^{ \pm}$and $u_{1, R}^{ \pm}$.

Then, 5.2 can easily be deduced by writing,

$$
R_{1}(z) h D_{x}=\left(I+R_{1}(z)\left(I+z-V_{1}\right)\right) h D_{x}\left(1-h^{2} \Delta\right)^{-1}
$$

(and the analogous formula for $R_{2}^{ \pm}(z)$ ), and by using (5.1) and the fact that $\left\|h D_{x}\left(1-h^{2} \Delta\right)^{-1}\right\|=\mathcal{O}(1)$.

\section{Function spaces}

In order to estimate in a systematic way the various integrals that are involved in the expressions of $r_{0}(t, \phi, h), r_{1}(t, \phi, h)$ and $r_{2}(t, \phi, h)$, we introduce several function spaces that, in some way, are related to the behavior (both semiclassical and at infinity in $x$ ) of the global WKB solutions of the scalar problems.

We set,

$$
\begin{aligned}
& m_{0}(x)=m_{0}(x ; h):=\min \left(h^{-1 / 6},|x|^{-1 / 4}\right) ; \\
& m_{*}(x)=m_{*}(x ; h):=\min \left(h^{-1 / 6},\left|x-x^{*}\right|^{-1 / 4}\right) .
\end{aligned}
$$

We define the space $\mathcal{F}_{1}\left(I_{L}\right)$ as the space of $h$-dependent smooth functions $u=u(x ; h)$ on $I_{L}$ for which, for any $\delta>0$ small enough and for any $k \geq 0$, there exists a constant $c=c_{k, \delta}>0$ such that,

- On $\left(-\infty, x^{*}-\delta\right],\left(h D_{x}\right)^{k} u(x ; h)=\mathcal{O}\left(e^{-c|x| / h}\right)$;

- On $\left[x^{*}-\delta, x^{*}\right],\left(h D_{x}\right)^{k} u(x ; h)=\mathcal{O}\left(m_{*}(x) e^{-c\left|x-x^{*}\right|^{3 / 2} / h}\right)$;

- On $\left[x^{*}, x^{*}+\delta\right],\left(h D_{x}\right)^{k} u(x ; h)=\mathcal{O}\left(m_{*}(x)\right)$; 
- On $\left[x^{*}+\delta,-\delta\right],\left(h D_{x}\right)^{k} u(x ; h)=\mathcal{O}(1)$;

- On $[-\delta, 0],\left(h D_{x}\right)^{k} u(x ; h)=\mathcal{O}\left(m_{0}(x)\right)$.

We also define the space $\mathcal{F}_{2}\left(I_{L}\right)$ as the space of $h$-dependent smooth functions $u=u(x ; h)$ on $I_{L}$ for which, for any $\delta>0$ small enough and for any $k \geq 0$, there exists a constant $c=c_{k, \delta}>0$ such that,

- On $(-\infty,-\delta],\left(h D_{x}\right)^{k} u(x ; h)=\mathcal{O}\left(e^{-c|x| / h}\right)$;

- On $[-\delta, 0],\left(h D_{x}\right)^{k} u(x ; h)=\mathcal{O}\left(m_{0}(x) e^{-c|x|^{3 / 2} / h}\right)$.

Analogously, we define the space $\mathcal{F}_{1}\left(I_{R}^{ \pm}\right)$as the space of $h$-dependent smooth functions $u=u(x ; h)$ on $I_{R}^{ \pm}$for which, for any $\delta>0$ and for any $k \geq 0$, there exists a constant $c=c_{k, \delta}>0$ such that,

- On $[0, \delta],\left(h D_{x}\right)^{k} u(x ; h)=\mathcal{O}\left(m_{0}(x) e^{-c|x|^{3 / 2} / h}\right)$;

- On $[\delta,+\infty),\left(h D_{x}\right)^{k} u(x ; h)=\mathcal{O}\left(e^{-c|x| / h}\right)$.

Finally, we define the space $\mathcal{F}_{2}\left(I_{R}^{ \pm}\right)$as the space of $h$-dependent smooth functions $u=u(x ; h)$ on $I_{R}^{ \pm}$for which, for any $\delta>0$ and for any $k \geq 0$, there exist two constants $c=c_{k, \delta}>0$ and $C=C_{k, \delta}>0$ such that,

- On $[0, \delta],\left(h D_{x}\right)^{k} u(x ; h)=\mathcal{O}\left(m_{0}(x)\right)$;

- On $I_{R}^{ \pm} \cap\{\delta \leq \operatorname{Re} x \leq C\},\left(h D_{x}\right)^{k} u(x ; h)=\mathcal{O}(1)$;

- On $I_{R}^{ \pm} \cap\{\operatorname{Re} x \geq C\},\left(h D_{x}\right)^{k} u(x ; h)=\mathcal{O}\left(e^{-c|x| / h}\right)$.

For $j, k \in\{1,2\}$, we also denote by $\mathcal{F}_{j}\left(I_{L}\right) \cap \mathcal{F}_{k}\left(I_{R}^{ \pm}\right)$the space of $h$-dependent functions $\varphi$ defined on $I_{L} \cup I_{R}^{ \pm}$(not necessarily smooth at 0 ), such that $\varphi_{L} \in \mathcal{F}_{j}\left(I_{L}\right)$ and $\varphi_{R} \in \mathcal{F}_{k}\left(I_{R}^{ \pm}\right)$. Of course, if such a function $\varphi$ is smooth at 0 , then, for any $\ell \geq 0$, one also has $\left(h D_{x}\right)^{\ell} \varphi \in \mathcal{F}_{j}\left(I_{L}\right) \cap \mathcal{F}_{k}\left(I_{R}^{ \pm}\right)$.

In particular, for any $z \in \mathcal{D}_{h}\left(C_{0}\right)$, we can see,

$$
\begin{array}{lll}
u_{1, L}^{-}(z) \in h^{1 / 6} \mathcal{F}_{1}\left(I_{L}\right) & ; & u_{1, R}^{-}(z) \in h^{1 / 6} \mathcal{F}_{1}\left(I_{R}^{ \pm}\right) ; \\
u_{2, L}^{-}(z) \in h^{1 / 6} \mathcal{F}_{2}\left(I_{L}\right) & ; & u_{2, R}^{\mp}(z) \in h^{1 / 6} \mathcal{F}_{2}\left(I_{R}^{ \pm}\right),
\end{array}
$$

and also, since $\varphi_{0} \sim h^{-1 / 6} u_{1, L}^{-}\left(\lambda_{0}\right)$ on $\mathbb{R}_{-}$, and $\varphi_{0} \sim h^{-1 / 6} u_{1, R}^{-}\left(\lambda_{0}\right)$ on $I_{R}^{ \pm}$,

$$
\varphi_{0} \in \mathcal{F}_{1}\left(I_{L}\right) \cap \mathcal{F}_{1}\left(I_{R}^{ \pm}\right) \cap C^{\infty},
$$

where $C^{\infty}$ stands for $C^{\infty}\left(I_{L} \cup I_{R}^{ \pm}\right)$, and just means that $\varphi_{0}$ is smooth at 0 , too.

We have (dropping the $z$-dependence), 
Proposition 6.1. The following inclusions hold:

$$
\begin{aligned}
& K_{1, L}\left(\mathcal{F}_{1}\left(I_{L}\right)\right) \subset h^{-1} \mathcal{F}_{1}\left(I_{L}\right) \quad ; \quad K_{1, L}\left(\mathcal{F}_{2}\left(I_{L}\right)\right) \subset h^{-2 / 3} \mathcal{F}_{1}\left(I_{L}\right) ; \\
& K_{2, L}\left(\mathcal{F}_{1}\left(I_{L}\right)\right) \subset h^{-2 / 3} \mathcal{F}_{1}\left(I_{L}\right) \quad ; \quad K_{2, L}\left(\mathcal{F}_{2}\left(I_{L}\right)\right) \subset h^{-2 / 3} \mathcal{F}_{2}\left(I_{L}\right),
\end{aligned}
$$

and,

$$
\begin{aligned}
K_{1, R}\left(\mathcal{F}_{1}\left(I_{R}^{ \pm}\right)\right) \subset h^{-2 / 3} \mathcal{F}_{1}\left(I_{R}^{ \pm}\right) & ; & K_{1, R}\left(\mathcal{F}_{2}\left(I_{R}^{ \pm}\right)\right) \subset h^{-2 / 3} \mathcal{F}_{2}\left(I_{R}^{ \pm}\right) \\
K_{2, R}^{ \pm}\left(\mathcal{F}_{1}\left(I_{R}^{ \pm}\right)\right) \subset h^{-2 / 3} \mathcal{F}_{2}\left(I_{R}^{ \pm}\right) & ; & K_{2, R}^{ \pm}\left(\mathcal{F}_{2}\left(I_{R}^{ \pm}\right)\right) \subset h^{-1} \mathcal{F}_{2}\left(I_{R}^{ \pm}\right) .
\end{aligned}
$$

Proof. See Appendix 1.

Remark 6.2. As an immediate consequence of the definitions of $\mathcal{F}_{j}\left(I_{L}\right)$ and $\mathcal{F}_{j}\left(I_{R}^{ \pm}\right)(j=1,2)$, we have

- If $v \in \mathcal{F}_{1}\left(I_{L}\right)$, then $\|v\|_{L^{2}\left(I_{L}\right)}=\mathcal{O}(1)$;

- If $v \in \mathcal{F}_{2}\left(I_{L}\right)$, then $\|v\|_{L^{2}\left(I_{L}\right)}=\mathcal{O}\left(h^{\frac{1}{6}}\right)$;

- If $v \in \mathcal{F}_{1}\left(I_{R}^{ \pm}\right)$, then $\|v\|_{L^{2}\left(I_{R}^{ \pm}\right)}=\mathcal{O}\left(h^{\frac{1}{6}}\right)$;

- If $v \in \mathcal{F}_{2}\left(I_{R}^{ \pm}\right)$, then $\|v\|_{L^{2}\left(I_{R}^{ \pm}\right)}=\mathcal{O}(1)$,

uniformly as $h \rightarrow 0_{+}$.

Proposition 6.3. For $z \in \gamma_{-}$, one has,

$$
\begin{aligned}
& \left|\alpha_{L, L}(z)\right|+\left|\alpha_{R, L}(z)\right|=\mathcal{O}\left(h^{-7 / 6}\right) \text { on } \mathcal{F}_{1}\left(I_{L}\right) ; \\
& \left|\beta_{L, L}^{ \pm}(z)\right|+\left|\beta_{R, L}^{ \pm}(z)\right|=\mathcal{O}\left(h^{-5 / 6}\right) \text { on } \mathcal{F}_{1}\left(I_{L}\right) ; \\
& \left|\alpha_{L, L}(z)\right|+\left|\alpha_{R, L}(z)\right|=\mathcal{O}\left(h^{-5 / 6}\right) \text { on } \mathcal{F}_{2}\left(I_{L}\right) ; \\
& \left|\beta_{L, L}^{ \pm}(z)\right|+\left|\beta_{R, L}^{ \pm}(z)\right|=\mathcal{O}\left(h^{-5 / 6}\right) \text { on } \mathcal{F}_{2}\left(I_{L}\right) ; \\
& \left|\alpha_{L, R}(z)\right|+\left|\alpha_{R, R}(z)\right|=\mathcal{O}\left(h^{-5 / 6}\right) \text { on } \mathcal{F}_{1}\left(I_{R}^{ \pm}\right) ; \\
& \left|\beta_{L, R}^{ \pm}(z)\right|+\left|\beta_{R, R}^{ \pm}(z)\right|=\mathcal{O}\left(h^{-5 / 6}\right) \text { on } \mathcal{F}_{1}\left(I_{R}^{ \pm}\right) ; \\
& \left|\alpha_{L, R}(z)\right|+\left|\alpha_{R, R}(z)\right|=\mathcal{O}\left(h^{-5 / 6}\right) \text { on } \mathcal{F}_{2}\left(I_{R}^{ \pm}\right) ; \\
& \left|\beta_{L, R}^{ \pm}(z)\right|+\left|\beta_{R, R}^{ \pm}(z)\right|=\mathcal{O}\left(h^{-7 / 6}\right) \text { on } \mathcal{F}_{2}\left(I_{R}^{ \pm}\right) .
\end{aligned}
$$

Proof. We use Remark 5.2 . Since $u_{1, L}^{-} \in h^{\frac{1}{6}} \mathcal{F}_{1}\left(I_{L}\right)$, for $\varphi \in \mathcal{F}_{1}\left(I_{L}\right)$ we have,

$$
\left|\alpha_{L, L}(z)[\varphi]\right|+\left|\alpha_{R, R}(z)[v]\right|=\mathcal{O}\left(h^{-\frac{4}{3}}\right)\left\|u_{1, L}^{-}\right\|_{L^{2}\left(I_{L}\right)}\|\varphi\|_{L^{2}\left(I_{L}\right)}=\mathcal{O}\left(h^{-\frac{4}{3}+\frac{1}{6}}\right) .
$$

Since $u_{2, L}^{-}$is exponentially concentrated at $x=0$, we also have,

$$
\left|\beta_{L, R}^{ \pm}(z)[\varphi]\right|+\left|\beta_{R, R}^{ \pm}(z)[\varphi]\right|=\mathcal{O}\left(h^{-\frac{4}{3}}\right) \int_{0}^{\delta} \frac{h^{\frac{1}{6}} e^{-c t^{3 / 2} / h}}{\sqrt{t}} d t+\mathcal{O}\left(e^{-c / h}\right),
$$


with $\delta>0$ arbitrarily small, and $c=c(\delta)>0$. Hence, after the change of variable $t \mapsto h^{\frac{2}{3}} t$, we find,

$$
\mid \beta_{L, R}^{ \pm}(z)\left[\varphi \left[|+| \beta_{R, R}^{ \pm}(z)[\varphi] \mid=\mathcal{O}\left(h^{-\frac{5}{6}}\right) .\right.\right.
$$

The other estimates follow along the same lines.

\section{Proposition 6.4.}

$$
R_{2}^{ \pm}(z)\left(\mathcal{F}_{1}\left(I_{L}\right) \cap \mathcal{F}_{1}\left(I_{R}^{ \pm}\right)\right) \subset h^{-2 / 3}\left(\mathcal{F}_{1}\left(I_{L}\right) \cap \mathcal{F}_{2}\left(I_{R}^{ \pm}\right)\right) .
$$

Proof. Let $\varphi \in \mathcal{F}_{1}\left(I_{L}\right) \cap \mathcal{F}_{1}\left(I_{R}^{ \pm}\right)$. By Proposition 5.1(iii), om $I_{L}$ we have,

$$
R_{2}^{ \pm}(z) \varphi \in K_{2, L}(z)\left(\mathcal{F}_{1}\left(I_{L}\right)\right)+\beta_{L}^{ \pm}(z)[\varphi] h^{\frac{1}{6}} \mathcal{F}_{2}\left(I_{L}\right),
$$

and therefore, using Propositions 6.1 and Remark 5.2

$$
R_{2}^{ \pm}(z) \varphi \in h^{-\frac{2}{3}} \mathcal{F}_{1}\left(I_{L}\right)+h^{-\frac{5}{6}+\frac{1}{6}} \mathcal{F}_{2}\left(I_{L}\right) \subset h^{-\frac{2}{3}} \mathcal{F}_{1}\left(I_{L}\right) .
$$

In the same way, by Proposition 5.1(iv), om $I_{R}^{ \pm}$we have,

$$
R_{2}^{ \pm}(z) \varphi \in K_{2, R}^{ \pm}(z)\left(\mathcal{F}_{1}\left(I_{R}^{ \pm}\right)\right)+\beta_{R}^{ \pm}(z)[\varphi] h^{\frac{1}{6}} \mathcal{F}_{2}\left(I_{R}^{ \pm}\right),
$$

and thus,

$$
R_{2}^{ \pm}(z) \varphi \in h^{-\frac{2}{3}} \mathcal{F}_{2}\left(I_{R}^{ \pm}\right)+h^{-\frac{5}{6}+\frac{1}{6}} \mathcal{F}_{2}\left(I_{R}^{ \pm}\right)=h^{-\frac{2}{3}} \mathcal{F}_{2}\left(I_{R}^{ \pm}\right) .
$$

As an immediate consequence of this proposition, if we set,

$$
\mathcal{H}_{0}^{ \pm}:=\mathcal{F}_{1}\left(I_{L}\right) \cap \mathcal{F}_{2}\left(I_{R}^{ \pm}\right),
$$

we have,

\section{Corollary 6.5.}

$$
R_{2}^{ \pm}(z) W^{*} \varphi_{0} \in h^{-2 / 3} \mathcal{H}_{0}^{ \pm} \cap C^{\infty}
$$

Finally, setting

$$
M_{ \pm}(z):=h^{2} R_{2}^{ \pm}(z) W^{*} R_{1}(z) W
$$

we have,

\section{Proposition 6.6.}

$$
M_{ \pm}(z)\left(\mathcal{H}_{0}^{ \pm} \cap C^{\infty}\right) \subset h^{1 / 3} \mathcal{H}_{0}^{ \pm} \cap C^{\infty} .
$$

In particular, for any $\ell \geq 1$,

$$
M_{ \pm}(z)^{\ell} R_{2}^{ \pm}(z) W^{*} \varphi_{0} \in h^{(\ell-2) / 3} \mathcal{H}_{0}^{ \pm} .
$$


Proof. By the same procedure as in the proof of Proposition 6.4, we see,

$$
R_{1}(z)\left(\mathcal{H}_{0}^{ \pm}\right) \subset h^{-1} \mathcal{F}_{1}\left(I_{L}\right) \cap\left[h^{-\frac{2}{3}} \mathcal{F}_{2}\left(I_{R}^{ \pm}\right)+h^{-1} \mathcal{F}_{1}\left(I_{R}^{ \pm}\right)\right]
$$

and also,

$$
R_{2}^{ \pm}(z)\left(\mathcal{F}_{1}\left(I_{L}\right) \cap h^{\frac{1}{3}} \mathcal{F}_{2}\left(I_{R}^{ \pm}\right)\right) \subset h^{-\frac{2}{3}} \mathcal{H}_{0}^{ \pm} .
$$

Since, with our definitions, we have,

$$
\begin{aligned}
h^{-1} \mathcal{F}_{1}\left(I_{L}\right) \cap & {\left[h^{-\frac{2}{3}} \mathcal{F}_{2}\left(I_{R}^{ \pm}\right)+h^{-1} \mathcal{F}_{1}\left(I_{R}^{ \pm}\right)\right] } \\
& =\left[h^{-1} \mathcal{F}_{1}\left(I_{L}\right) \cap h^{-\frac{2}{3}} \mathcal{F}_{2}\left(I_{R}^{ \pm}\right)\right]+\left[h^{-1} \mathcal{F}_{1}\left(I_{L}\right) \cap h^{-1} \mathcal{F}_{1}\left(I_{R}^{ \pm}\right)\right]
\end{aligned}
$$

we deduce,

$$
R_{2}^{ \pm}(z) W^{*} R_{1}(z)\left(\mathcal{H}_{0}^{ \pm}\right) \subset h^{-\frac{5}{3}} \mathcal{H}_{0}^{ \pm}+h^{-1} R_{2}^{ \pm}(z)\left(\mathcal{F}_{1}\left(I_{L}\right) \cap \mathcal{F}_{1}\left(I_{R}^{ \pm}\right)\right)
$$

that is, by Proposition 6.4 .

$$
R_{2}^{ \pm}(z) W^{*} R_{1}(z)\left(\mathcal{H}_{0}^{ \pm}\right) \subset h^{-\frac{5}{3}} \mathcal{H}_{0}^{ \pm} .
$$

Since in addition $W\left(\mathcal{H}_{0}^{ \pm} \cap C^{\infty}\right) \subset \mathcal{H}_{0}^{ \pm} \cap C^{\infty}$, and $R_{1}(z), R_{2}^{ \pm}(z)$ preserve the regularity at 0 , the result follows.

\section{Estimates ON $r_{2}(t, \phi, h)$}

We first show,

Lemma 7.1. For any $u \in \mathcal{H}_{0}^{ \pm}$, one has,

$$
\begin{gathered}
\left\langle u, W^{*} \varphi_{0}\right\rangle_{L^{2}\left(I_{L}\right)}=\mathcal{O}(1) ; \\
\left\langle u, W^{*} \varphi_{0}\right\rangle_{L^{2}\left(I_{R}^{ \pm}\right)}=\mathcal{O}\left(h^{1 / 3}\right) .
\end{gathered}
$$

Proof. Since $W^{*} \varphi_{0} \in \mathcal{F}_{1}\left(I_{L}\right) \cap \mathcal{F}_{1}\left(I_{R}^{ \pm}\right)$, the first estimate is immediate, while for the second one, thanks to the exponantial localization near 0 of $\left.\varphi_{0}\right|_{I_{R}^{ \pm}}$, we can write,

$$
\left\langle u, W^{*} \varphi_{0}\right\rangle_{L^{2}\left(I_{R}^{ \pm}\right)}=\mathcal{O}(1) \int_{0}^{\delta} \frac{e^{-c t^{3 / 2} / h}}{\sqrt{t}} d t+\mathcal{O}\left(e^{-c / h}\right),
$$

(with $\delta>0$ sufficiently small and $c=c(\delta)>0$ ), and the result follows.

Then, we have,

Proposition 7.2. One has,

$$
r_{2}(t, \phi, h)=\mathcal{O}\left(h\langle h t\rangle^{-\infty}\right) .
$$


Proof. We must prove that, for any $k \geq 0$, we have $r_{2}(t, \phi, h)=\mathcal{O}\left(h\langle h t\rangle^{-k}\right)$. By Corollary 5.3, we already know that there exists a constant $C>0$ such that $\left|T_{\ell}(z)\right| \leq C^{\ell} h^{-1+(\ell-1) / 6}$ uniformly with respect to $h$ small enough. Therefore, for any $L_{0} \geq 1$,

$$
r_{2}(t, \phi, h)=\frac{h^{2}}{2 i \pi} \sum_{\ell=2}^{L_{0}} \int_{\gamma_{-}} \frac{e^{-i t z} g(\operatorname{Re} z)}{\left(\lambda_{0}-z\right)^{2}} T_{\ell}(z) d z+\mathcal{O}\left(h^{\left(L_{0}-1\right) / 6}\right) .
$$

In particular,

$$
r_{2}(t, \phi, h)=\frac{h^{2}}{2 i \pi} \sum_{\ell=2}^{6} \int_{\gamma_{-}} \frac{e^{-i t z} g(\operatorname{Re} z)}{\left(\lambda_{0}-z\right)^{2}} T_{\ell}(z) d z+\mathcal{O}(h) .
$$

Moreover, using Proposition 6.6 and Lemma 7.1, for any $\ell \in\{2, \ldots, 6\}$, we have,

$$
T_{\ell}(z)=\mathcal{O}\left(h^{(\ell-2) / 3}\right)
$$

and thus,

$$
\frac{h^{2}}{2 i \pi} \sum_{\ell=2}^{6} \int_{\gamma_{-}} \frac{e^{-i t z} g(\operatorname{Re} z)}{\left(\lambda_{0}-z\right)^{2}} T_{\ell}(z) d z=\mathcal{O}(h) .
$$

so that the result for $k=0$ follows. The result for $k \geq 1$ is obtained by using that $e^{-i t z}=(1+h t)^{-k}\left(1+i h \partial_{z}\right)^{k}\left(e^{-i t z}\right)$ and by making $k$ integrations by parts. Each derivative $h \partial_{z}$ that falls on $g(\operatorname{Re} z)\left(\lambda_{0}-z\right)^{-2}$, doesn't make us lose anything in the estimate. If instead it falls down on $T_{\ell}(z)$, we need the following,

Lemma 7.3. For any $k, \ell \geq 1$, one has,

$$
h^{k} \partial_{z}^{k} T_{\ell}(z)=\mathcal{O}\left(h^{\frac{\ell-2}{3}}\right) .
$$

Moreover, for any $k, \ell \geq 1$, there exists a constant $C_{k}$ such that, for all $\ell \geq 1$,

$$
\left\|h^{k} \partial_{z}^{k}\left(M_{ \pm}(z)^{\ell}\right)\right\| \leq C_{k}^{\ell} h^{\frac{\ell}{6}}
$$

Proof. Going back to the construction of the functions $u_{j, L}^{ \pm}(z, x)$ and $u_{j, R}^{ \pm}(z, x)$ (see [FMW1, Appendix]), we start by observing that they all are of the form $\left(\partial_{x} \xi(x, z)\right)^{-1 / 2} f_{z}\left(h^{-2 / 3} \xi(x, z)\right)$, where $x \mapsto \xi(x, z)$ is a global analytic change of variable that depends analytically on $z$, and $f_{z}$ is solution to a Volterra problem of the type,

$$
f_{z}=F+K_{z} f_{z}
$$

with $z \mapsto K_{z}$ holomorphic, and the norm of $K_{z}$ (and of all its holomorphic derivatives with respect to $z$ ) is small as $h$ tends to 0 (here, $K_{z}$ acts on a space continuous functions with some specific growth at infinity depending 
on the choice of $F$ ). In addition, the function $F$ appearing in $(7.5)$ is always taken in the set $\{\mathrm{Ai}, \mathrm{Bi}, \check{\mathrm{Ai}}, \check{\mathrm{Bi}}\}$. It results that $z \mapsto f_{z}$ is holomorphic, too, and that, for all $k, \ell, \partial_{z}^{k} \partial_{x}^{\ell} f_{z}$ growths at most as $\sum_{m=0}^{\ell}\left|F^{(\ell)}\right|$ at infinity.

Then, considering the function $u_{z}(x):=f_{z}\left(h^{-2 / 3} \xi(x, z)\right)$, we deduce,

$$
\partial_{z}^{k} u_{z}(x)=\mathcal{O}\left(h^{-2 k / 3} \sum_{m=0}^{\ell}\left|F^{(\ell)}\left(h^{-2 / 3} \xi(x)\right)\right|\right) .
$$

Now, because of the behavior at infinity of the Airy functions, and of the possible choices of the function $F$, we see that,

$$
\left.\sum_{m=0}^{\ell}\left|F^{(\ell)}(t)\right|\right)=\mathcal{O}\left(\langle t\rangle^{\ell / 2} F_{0}(t)\right),
$$

where $F_{0}$ reflects the behavior of $F$ at infinity, that is, $F_{0}(t)=\langle t\rangle^{-1 / 4} e^{ \pm \frac{2}{3}|t|^{\frac{3}{2}}}$ if $F$ has an exponential behavior, and $F_{0}(t)=\langle t\rangle^{-1 / 4}$ if $F$ oscillates at infinity. Therefore, we obtain,

$$
\sum_{m=0}^{\ell}\left|F^{(\ell)}\left(h^{-2 / 3} \xi(x)\right)\right|=\mathcal{O}\left(h^{-k / 3}\right) F_{0}\left(h^{-2 / 3} \xi(x)\right),
$$

and thus,

$$
\left.\partial_{z}^{k} u_{z}(x)=\mathcal{O}\left(h^{-k}\right) F_{0}\left(h^{-2 / 3} \xi(x)\right)\right)
$$

In particular, for $j=1,2, S \in\{L, R\}$, and any $k \geq 0$, the function $h^{k} \partial_{z}^{k} u_{j, S}^{ \pm}$ has the same behavior (both semiclassical and at infinity) as the function $u_{j, S}^{ \pm}$itself.

As a consequence, considering the operator $h^{k} \partial_{z}^{k}\left(M_{ \pm}(z)\right)^{\ell}$, we see that it is a sum of $\ell^{k}$ products of $\ell$ factors, each one of them being of the same type as $M_{ \pm}(z)$, and $(7.4)$ follows.

For the same reasons, we also have,

$$
h^{k} \partial_{z}^{k}\left(M_{ \pm}(z)\right)^{\ell}\left(\mathcal{H}_{0}^{ \pm}\right) \subset h^{\ell / 3} \mathcal{H}_{0}^{ \pm},
$$

and

$$
h^{k} \partial_{z}^{k} R_{2}^{ \pm}(z) W^{*} \varphi_{0} \in \mathcal{H}_{0}^{ \pm},
$$

so that 7.3 follows, too.

Using Lemma 7.3 and making integrations by parts in the expression of $r_{2}$ given in (3.10), Proposition 7.2 follows. 
8. Estimates ON $r_{1}(t, \phi, h)$

Concerning $r_{1}(t, \phi, h)$, the same arguments of the previous section can be applied, but they lead to an estimate in $\mathcal{O}\left(h^{2 / 3}\langle h t\rangle^{-\infty}\right)$ only. Let us prove that actually, we have,

Proposition 8.1. One has,

$$
r_{1}(t, \phi, h)=\mathcal{O}\left(h\langle h t\rangle^{-\infty}\right) .
$$

Proof. In view of Proposition 6.6 and Lemma 7.1, we can write,

$$
T_{1}(z)=\left\langle\left(M_{+}(z) R_{2}^{+}(z)-M_{-}(z) R_{2}^{-}(z)\right) W^{*} \varphi_{0}, W^{*} \varphi_{0}\right\rangle_{L^{2}\left(I_{L}\right)}+\mathcal{O}(1),
$$

that is, by Proposition 5.1

$$
\begin{aligned}
T_{1}(z)= & h^{2}\left\langle\left(K_{2, L}+B_{L}^{+}\right) W^{*} R_{1} W R_{2}^{+} W^{*} \varphi_{0}, W^{*} \varphi_{0}\right\rangle_{L^{2}\left(I_{L}\right)} \\
& -h^{2}\left\langle\left(K_{2, L}+B_{L}^{-}\right) W^{*} R_{1} W R_{2}^{-} W^{*} \varphi_{0}, W^{*} \varphi_{0}\right\rangle_{L^{2}\left(I_{L}\right)}+\mathcal{O}(1),
\end{aligned}
$$

where we have omited the dependence in $z$ of the various operators, and where we have set,

$$
B_{L}^{ \pm} \varphi:=\beta_{L}^{ \pm}(\varphi) u_{2, L}^{-}
$$

Let us first prove,

Lemma 8.2. For all $z \in \gamma_{-}$, one has,

$$
\begin{aligned}
& R_{1}(z) W R_{2}^{ \pm}(z) W^{*} \varphi_{0} \\
& \quad \in h^{-5 / 3} \mathcal{F}_{1}\left(I_{L}\right) \cap\left(h^{-4 / 3} \mathcal{F}_{2}\left(I_{R}^{ \pm}\right)+h^{-5 / 3} \mathcal{F}_{1}\left(I_{R}^{ \pm}\right)\right) .
\end{aligned}
$$

Proof. By Proposition 6.4, we already know that $\psi_{ \pm}:=W R_{2}^{ \pm}(z) W^{*} \varphi_{0}$ is in $h^{-\frac{2}{3}} \mathcal{H}_{0}^{ \pm}$. Then, the result directly follows from 6.4 .

We deduce from the previous lemma and from Lemma ?? that we have,

$$
\beta_{L}^{ \pm}\left(W^{*} R_{1} \psi_{ \pm}\right)=\mathcal{O}\left(h^{-15 / 6}\right),
$$

and thus,

$$
B_{L}^{ \pm} W^{*} R_{1} \psi_{ \pm} \in h^{-7 / 3} \mathcal{F}_{2}\left(I_{L}\right) .
$$

As a consequence, by an elementary computation we obtain (using also (6.2) $)$

$$
h^{2}\left\langle B_{L}^{ \pm} W^{*} R_{1} \psi_{ \pm}, W^{*} \varphi_{0}\right\rangle_{L^{2}\left(I_{L}\right)}=\mathcal{O}\left(h^{2-\frac{7}{3}}\right) \int_{0}^{\delta} \frac{e^{-c x^{3 / 2} / h}}{\sqrt{x}} d x+\mathcal{O}\left(e^{-\delta^{\prime} / h}\right),
$$


(where $\delta, \delta^{\prime}$ and $c$ are positive constants), and thus,

$$
h^{2}\left\langle B_{L}^{ \pm} W^{*} R_{1} \psi_{ \pm}, W^{*} \varphi_{0}\right\rangle_{L^{2}\left(I_{L}\right)}=\mathcal{O}(1) .
$$

Therefore, going back to (8.1), we deduce,

$$
\begin{aligned}
T_{1}(z)= & h^{2}\left\langle K_{2, L} W^{*} R_{1} W R_{2}^{+} W^{*} \varphi_{0}, W^{*} \varphi_{0}\right\rangle_{L^{2}\left(I_{L}\right)} \\
& -h^{2}\left\langle K_{2, L} W^{*} R_{1} W R_{2}^{-} W^{*} \varphi_{0}, W^{*} \varphi_{0}\right\rangle_{L^{2}\left(I_{L}\right)}+\mathcal{O}(1),
\end{aligned}
$$

that is,

$$
\begin{aligned}
T_{1}(z)= & h^{2}\left\langle K_{2, L} W^{*}\left(K_{1, L}+A_{L}\right) W R_{2}^{+} W^{*} \varphi_{0}, W^{*} \varphi_{0}\right\rangle_{L^{2}\left(I_{L}\right)} \\
& -h^{2}\left\langle K_{2, L} W^{*}\left(K_{1, L}+A_{L}\right) W R_{2}^{-} W^{*} \varphi_{0}, W^{*} \varphi_{0}\right\rangle_{L^{2}\left(I_{L}\right)}+\mathcal{O}(1),
\end{aligned}
$$

where this time we have set,

$$
A_{L}:=A_{L L}+A_{L R},
$$

with

$$
A_{L L}(\varphi):=\alpha_{L L}\left(\varphi_{L}\right) u_{1, L}^{-} \quad ; \quad A_{L R}(\varphi):=\alpha_{L R}\left(\varphi_{R}\right) u_{1, L}^{-} .
$$

In particular, setting,

$$
B_{R}^{ \pm} \varphi:=\beta_{R}^{ \pm}(\varphi) u_{2, R}^{\mp}
$$

we can rewrite (8.4) as,

$$
\begin{aligned}
T_{1}(z)= & h^{2}\left\langle K_{2, L} W^{*}\left(K_{1, L}+A_{L L}\right) W\left(K_{2, L}+B_{L}^{+}\right) W^{*} \varphi_{0}, W^{*} \varphi_{0}\right\rangle_{L^{2}\left(I_{L}\right)} \\
& -h^{2}\left\langle K_{2, L} W^{*}\left(K_{1, L}+A_{L L}\right) W\left(K_{2, L}+B_{L}^{-}\right) W^{*} \varphi_{0}, W^{*} \varphi_{0}\right\rangle_{L^{2}\left(I_{L}\right)} \\
& +h^{2}\left\langle K_{2, L} W^{*} A_{L R} W\left(K_{2, R}+B_{R}^{+}\right) W^{*} \varphi_{0}, W^{*} \varphi_{0}\right\rangle_{L^{2}\left(I_{L}\right)} \\
& -h^{2}\left\langle K_{2, L} W^{*} A_{L R} W\left(K_{2, R}+B_{R}^{-}\right) W^{*} \varphi_{0}, W^{*} \varphi_{0}\right\rangle_{L^{2}\left(I_{L}\right)}+\mathcal{O}(1),
\end{aligned}
$$

that is, after having eliminated the terms that cancel,

$$
\begin{aligned}
T_{1}(z)= & h^{2}\left\langle K_{2, L} W^{*}\left(K_{1, L}+A_{L L}\right) W\left(B_{L}^{+}-B_{L}^{-}\right) W^{*} \varphi_{0}, W^{*} \varphi_{0}\right\rangle_{L^{2}\left(I_{L}\right)} \\
& +h^{2}\left\langle K_{2, L} W^{*} A_{L R} W\left(B_{R}^{+}-B_{R}^{-}\right) W^{*} \varphi_{0}, W^{*} \varphi_{0}\right\rangle_{L^{2}\left(I_{L}\right)}+\mathcal{O}(1) .
\end{aligned}
$$

Using again Lemma ?? and (6.1), we find,

$$
W B_{L}^{ \pm} W^{*} \varphi_{0} \in h^{-2 / 3} \mathcal{F}_{2}\left(I_{L}\right) \quad ; \quad W B_{R}^{ \pm} W^{*} \varphi_{0} \in h^{-2 / 3} \mathcal{F}_{2}\left(I_{R}^{ \pm}\right),
$$

and thus also,

Therefore, by Proposition 6.1.

$$
\begin{aligned}
& W^{*} A_{L L} W B_{L}^{ \pm} W^{*} \varphi_{0} \in h^{-4 / 3} \mathcal{F}_{1}\left(I_{L}\right) ; \\
& W^{*} A_{L R} W B_{R}^{ \pm} W^{*} \varphi_{0} \in h^{-4 / 3} \mathcal{F}_{1}\left(I_{L}\right) .
\end{aligned}
$$

$$
\begin{aligned}
& K_{2, L} W^{*} A_{L L} W B_{L}^{ \pm} W^{*} \varphi_{0} \in h^{-2} \mathcal{F}_{1}\left(I_{L}\right) ; \\
& K_{2, L} W^{*} A_{L R} W B_{R}^{ \pm} W^{*} \varphi_{0} \in h^{-2} \mathcal{F}_{1}\left(I_{L}\right) .
\end{aligned}
$$

As a consequence, we obtain (with $S=L, R$ ),

$$
h^{2}\left\langle K_{2, L} W^{*} A_{L S} W B_{S}^{ \pm} W^{*} \varphi_{0}, W^{*} \varphi_{0}\right\rangle_{L^{2}\left(I_{L}\right)}=\mathcal{O}(1),
$$


and (8.5) reduces to,

$$
T_{1}(z)=h^{2}\left\langle K_{2, L} W^{*} K_{1, L} W\left(B_{L}^{+}-B_{L}^{-}\right) W^{*} \varphi_{0}, W^{*} \varphi_{0}\right\rangle_{L^{2}\left(I_{L}\right)}+\mathcal{O}(1) .
$$

Using (8.6) and, once more, Proposition 6.1. we obtain,

$$
K_{2, L} W K_{1, L} W^{*} B_{L}^{ \pm} W^{*} \varphi_{0} \in K_{2, L}\left(h^{-4 / 3} \mathcal{F}_{1}\left(I_{L}\right)\right) \subset h^{-2} \mathcal{F}_{1}\left(I_{L}\right),
$$

so that the same computations finally give us,

$$
T_{1}(z)=\mathcal{O}(1) .
$$

Hence, $r_{1}(t, \varphi, h)=\mathcal{O}(h)$ and Proposition 8.1 follows by the same arguments as in the previous section.

\section{Estimates ON $r_{0}(t, \phi, h)$}

By definition we have,

$$
\begin{aligned}
T_{0}(z)= & \left\langle\left(R_{2}^{+}(z)-R_{2}^{-}(z)\right) W^{*} \varphi_{0}, W^{*} \varphi_{0}\right\rangle_{L^{2}\left(I_{L}\right)} \\
& +\left\langle R_{2}^{+}(z) W^{*} \varphi_{0}, W^{*} \varphi_{0}\right\rangle_{L^{2}\left(I_{R}^{+}\right)}-\left\langle R_{2}^{-}(z) W^{*} \varphi_{0}, W^{*} \varphi_{0}\right\rangle_{L^{2}\left(I_{R}^{-}\right)},
\end{aligned}
$$

and thus, by Proposition 5.1 ,

$$
T_{0}(z)=T_{0,1}(z)+T_{0,2}(z)+T_{0,3}(z)
$$

with (using the same notations 8.2 as in the previous section),

$$
\begin{aligned}
& T_{0,1}(z)=\left\langle\left(B_{L}^{+}(z)-B_{L}^{-}(z)\right) W^{*} \varphi_{0}, W^{*} \varphi_{0}\right\rangle_{L^{2}\left(I_{L}\right)} \\
& T_{0,2}(z)=\left\langle B_{R}^{+}(z) W^{*} \varphi_{0}, W^{*} \varphi_{0}\right\rangle_{L^{2}\left(I_{R}^{+}\right)}-\left\langle B_{R}^{-}(z) W^{*} \varphi_{0}, W^{*} \varphi_{0}\right\rangle_{L^{2}\left(I_{R}^{-}\right)} ; \\
& T_{0,3}(z)=\left\langle K_{2, R}^{+}(z) W^{*} \varphi_{0}, W^{*} \varphi_{0}\right\rangle_{L^{2}\left(I_{R}^{+}\right)}-\left\langle K_{2, R}^{-}(z) W^{*} \varphi_{0}, W^{*} \varphi_{0}\right\rangle_{L^{2}\left(I_{R}^{-}\right)} .
\end{aligned}
$$

We first show,

Proposition 9.1. Setting $\rho:=h^{-\frac{2}{3}} z$ and $\mu_{0}:=h^{-\frac{2}{3}} \lambda_{0}$, one has,

$$
\begin{aligned}
& \left\langle u_{2, L}^{-}(z), W^{*} \varphi_{0}\right\rangle_{L^{2}\left(I_{L}\right)}=4 a_{0}(0) c_{0} h^{\frac{1}{2}} A^{-}(\rho)+\mathcal{O}\left(h^{\frac{5}{6}}\right) ; \\
& \left\langle u_{2, R}^{-}(z), W^{*} \varphi_{0}\right\rangle_{L^{2}\left(I_{R}^{+}\right)}=\sqrt{2} a_{0}(0) c_{0} h^{\frac{1}{2}} e^{i \frac{\pi}{4}}\left(A^{+}(\rho)-i B^{+}(\rho)\right)+\mathcal{O}\left(h^{\frac{5}{6}}\right) ; \\
& \left\langle u_{2, R}^{+}(z), W^{*} \varphi_{0}\right\rangle_{L^{2}\left(I_{R}^{-}\right)}=2 \sqrt{2} a_{0}(0) c_{0} h^{\frac{1}{2}} e^{i \frac{\pi}{4}}\left(A^{+}(\rho)+i B^{+}(\rho)\right)+\mathcal{O}\left(h^{\frac{5}{6}}\right),
\end{aligned}
$$

with,

$$
\begin{aligned}
& A^{ \pm}(\rho):=\tau_{1}^{-\frac{1}{6}} \tau_{2}^{-\frac{1}{6}} \int_{\mathbb{R}_{ \pm}} \check{\mathrm{Ai}}\left(\tau _ { 2 } ^ { \frac { 1 } { 3 } } ( y + \frac { \rho } { \tau _ { 2 } } ) \mathrm { Ai } \left(\tau_{1}^{\frac{1}{3}}\left(y-\frac{\mu_{0}}{\tau_{1}}\right) d y\right.\right. \\
& B^{+}(\rho):=\tau_{1}^{-\frac{1}{6}} \tau_{2}^{-\frac{1}{6}} \int_{0}^{+\infty} \check{\mathrm{Bi}}\left(\tau _ { 2 } ^ { \frac { 1 } { 3 } } ( y + \frac { \rho } { \tau _ { 2 } } ) \mathrm { Ai } \left(\tau_{1}^{\frac{1}{3}}\left(y-\frac{\mu_{0}}{\tau_{1}}\right) d y .\right.\right.
\end{aligned}
$$

Moreover, the same formulas hold if $W^{*} \varphi_{0}$ is substituted by $\overline{W^{*} \varphi_{0}}$. 
Proof. The proof is similar to that of [FMW1, Proposition 5.3]. In practical, we cut the integral on $I_{L}$ into $\int_{-\infty}^{-\lambda h^{\frac{2}{3}}}+\int_{-\lambda h^{\frac{2}{3}}}^{0}$, and that on $I_{R}^{+}$into $\int_{0}^{\lambda h^{\frac{2}{3}}}+\int_{I_{R}^{+} \cap\left\{\operatorname{Re} x \geq \lambda h^{\frac{2}{3}}\right\}}^{0}$, where $\lambda=C \ln |h|$, with $C>0$ a large enough constant. Then, we use the exponential decay of $u_{2, L}^{-}(z)$ away from 0 on $\mathbb{R}_{-}$, and that of $W^{*} \varphi_{0}$ away from 0 on $I_{R}^{+}$, in order to estimate the integrals on $\left(-\infty,-\lambda h^{\frac{2}{3}}\right]$ and on $I_{R}^{+} \cap\left\{\operatorname{Re} x \geq \lambda h^{\frac{2}{3}}\right\}$, and finally, near 0 we replace $u_{2, L}^{-}(z), u_{2, R}^{-}(z)$ and $\varphi_{0}$ by their approximations in terms of Airy functions (see [FMW1, Appendix 2],

$$
\begin{aligned}
& u_{2, L}^{-}(z, x)=2\left(\xi_{2}^{\prime}\right)^{-\frac{1}{2}} \check{\mathrm{Ai}}\left(h^{-\frac{2}{3}} \xi_{2}\right)+\mathcal{O}(h) \\
& u_{2, R}^{-}(z, x)=\frac{1}{\sqrt{2}} e^{i \frac{\pi}{4}}\left(\xi_{2}^{\prime}\right)^{-\frac{1}{2}}\left(\check{\mathrm{Ai}}\left(h^{-\frac{2}{3}} \xi_{2}\right)-i \check{\mathrm{Bi}}\left(h^{-\frac{2}{3}} \xi_{2}\right)\right)+\mathcal{O}(h) ; \\
& u_{2, R}^{+}(z, x)=\sqrt{2} e^{i \frac{\pi}{4}}\left(\xi_{2}^{\prime}\right)^{-\frac{1}{2}}\left(\check{\mathrm{Ai}}\left(h^{-\frac{2}{3}} \xi_{2}\right)+i \check{\mathrm{Bi}}\left(h^{-\frac{2}{3}} \xi_{2}\right)\right)+\mathcal{O}(h) ; \\
& \varphi_{0}(x)=2 c_{0} h^{-\frac{1}{6}}\left(\xi_{1}^{\prime}\right)^{-\frac{1}{2}} \mathrm{Ai}\left(h^{-\frac{2}{3}} \xi_{1}\right)+\mathcal{O}(h),
\end{aligned}
$$

where $\xi_{1}=\xi_{1}(x)$ and $\xi_{2}(z, x)$ satisfy (see [FMW1, Section7]),

$$
\begin{aligned}
& h^{-\frac{2}{3}} \xi_{1}\left(h^{\frac{2}{3}} y\right)=\tau_{1}^{\frac{1}{3}}\left(y-\frac{\mu_{0}}{\tau_{1}}\right)+\mathcal{O}\left(h^{\frac{2}{3}}\right) \\
& h^{-\frac{2}{3}} \xi_{2}\left(z, h^{\frac{2}{3}} y\right)=\tau_{2}^{\frac{1}{3}}\left(y+\frac{\rho}{\tau_{1}}\right)+\mathcal{O}\left(h^{\frac{2}{3}}\right) .
\end{aligned}
$$

Then, we prove,

Proposition 9.2. Still with $\rho:=h^{-\frac{2}{3}} z$, one has,

$$
T_{0,1}(z)=8 i \pi h^{-\frac{1}{3}} c_{0}^{2} a_{0}(0)^{2}\left[A^{+}(\rho)+A^{-}(\rho)\right] A^{-}(\rho)+\mathcal{O}(1)
$$

$$
\begin{gathered}
T_{0,2}(z)=4 i \pi h^{-\frac{1}{3}} c_{0}^{2} a_{0}(0)^{2}\left[2 A^{+}(\rho) A^{-}(\rho)+A^{+}(\rho)^{2}-B^{+}(\rho)^{2}\right]+\mathcal{O}(1) \\
T_{0,3}(z)=4 i \pi h^{-\frac{1}{3}} c_{0}^{2} a_{0}(0)^{2}\left[A^{+}(\rho)^{2}+B^{+}(\rho)^{2}\right]+\mathcal{O}(1)
\end{gathered}
$$

Proof. Setting,

$$
\varphi_{1}:=W^{*} \varphi_{0}
$$

by definition we have,

$$
T_{0,1}=\left[\beta_{L, L}^{+}\left(\varphi_{1}\right)-\beta_{L, L}^{-}\left(\varphi_{1}\right)+\beta_{L, R}^{+}\left(\varphi_{1}\right)-\beta_{L, R}^{-}\left(\varphi_{1}\right)\right]\left\langle u_{2, L}^{-}, \varphi_{1}\right\rangle .
$$


Then, using Remark 5.2 and proceeding as in the proof of Proposition 9.1 we see,

$$
\begin{aligned}
\beta_{L, L}^{+}\left(\varphi_{1}\right)-\beta_{L, L}^{-}\left(\varphi_{1}\right) & =i \frac{\pi}{2} h^{-\frac{4}{3}}\left(1+\mathcal{O}\left(h^{\frac{1}{3}}\right)\right) \int_{-\infty}^{0} u_{2, L}^{-}(z, t) \varphi_{1}(t) d t \\
& =2 i \pi a_{0}(0) c_{0} A^{-}(\rho) h^{-\frac{5}{6}}+\mathcal{O}\left(h^{-\frac{1}{2}}\right) ; \\
\beta_{L, R}^{+}\left(\varphi_{1}\right) & =\frac{\pi}{\sqrt{2}} a_{0}(0) c_{0} e^{i \frac{\pi}{4}} h^{-\frac{5}{6}} \frac{2}{\sqrt{2}} e^{i \frac{\pi}{4}}\left(A^{+}(\rho)-i B^{+}(\rho)\right)+\mathcal{O}\left(h^{-\frac{1}{2}}\right) \\
& =i \pi a_{0}(0) c_{0}\left(A^{+}(\rho)-i B^{+}(\rho)\right) h^{-\frac{5}{6}}+\mathcal{O}\left(h^{-\frac{1}{2}}\right) ; \\
\beta_{L, R}^{-}\left(\varphi_{1}\right) & =-i \pi a_{0}(0) c_{0}\left(A^{+}(\rho)+i B^{+}(\rho)\right) h^{-\frac{5}{6}}+\mathcal{O}\left(h^{-\frac{1}{2}}\right) .
\end{aligned}
$$

Hence,

$$
\begin{aligned}
\beta_{L, L}^{+}\left(\varphi_{1}\right)-\beta_{L, L}^{-}\left(\varphi_{1}\right)+ & \beta_{L, R}^{+}\left(\varphi_{1}\right)-\beta_{L, R}^{-}\left(\varphi_{1}\right) \\
& =2 i \pi a_{0}(0) c_{0}\left(A^{-}(\rho)+A^{+}(\rho)\right) h^{-\frac{5}{6}}+\mathcal{O}\left(h^{-\frac{1}{2}}\right),
\end{aligned}
$$

which, together with Proposition 9.1, gives (9.5).

In the same way, we have,

$$
\begin{aligned}
& \beta_{R, L}^{+}\left(\varphi_{1}\right)=\frac{4 \pi}{\sqrt{2}} e^{i \frac{\pi}{4}} a_{0}(0) c_{0} A^{-}(\rho) h^{-\frac{5}{6}}+\mathcal{O}\left(h^{-\frac{1}{2}}\right) \\
& \beta_{R, R}^{+}\left(\varphi_{1}\right)=\frac{2 \pi}{\sqrt{2}} e^{i \frac{\pi}{4}} a_{0}(0) c_{0}\left(A^{+}(\rho)-i B^{+}(\rho)\right) h^{-\frac{5}{6}}+\mathcal{O}\left(h^{-\frac{1}{2}}\right) \\
& \beta_{R, L}^{-}\left(\varphi_{1}\right)=-\frac{2 \pi}{\sqrt{2}} e^{i \frac{\pi}{4}} a_{0}(0) c_{0} A^{-}(\rho) h^{-\frac{5}{6}}+\mathcal{O}\left(h^{-\frac{1}{2}}\right) \\
& \beta_{R, R}^{-}\left(\varphi_{1}\right)=-\frac{\pi}{\sqrt{2}} e^{i \frac{\pi}{4}} a_{0}(0) c_{0}\left(A^{+}(\rho)+i B^{+}(\rho)\right) h^{-\frac{5}{6}}+\mathcal{O}\left(h^{-\frac{1}{2}}\right) .
\end{aligned}
$$

and thus, by Proposition 9.1 ,

$$
\begin{aligned}
\left\langle B_{R}^{+} \varphi_{1}, \varphi_{1}\right\rangle_{L^{2}\left(I_{R}^{+}\right)}=2 i \pi a_{0}(0)^{2} c_{0}^{2}\left(2 A^{-}(\rho)\right. & \left.+A^{+}(\rho)-i B^{+}(\rho)\right) \\
& \times\left(A^{+}(\rho)-i B^{+}(\rho)\right) h^{-\frac{1}{3}}+\mathcal{O}(1) ; \\
\left\langle B_{R}^{-} \varphi_{1}, \varphi_{1}\right\rangle_{L^{2}\left(I_{R}^{-}\right)}=-2 i \pi a_{0}(0)^{2} c_{0}^{2}\left(2 A^{-}(\rho)\right. & \left.+A^{+}(\rho)+i B^{+}(\rho)\right) \\
& \times\left(A^{+}(\rho)+i B^{+}(\rho)\right) h^{-\frac{1}{3}}+\mathcal{O}(1) .
\end{aligned}
$$

Then, (9.6) follows by a straightforward computation.

Finally, concerning $T_{0,3}$, using the exponential decay of $\varphi_{1}$ on $I_{R}^{ \pm}$away from 0 , for any $\delta>0$ small enough we can write,

$$
T_{0,3}=\left\langle K_{2, R}^{+} \varphi_{1}-K_{2, R}^{-} \varphi_{1}, \varphi_{1}\right\rangle_{L^{2}(0, \delta)}+\mathcal{O}\left(e^{-c / h}\right),
$$


with $c=c(\delta)>0$ constant. Now, we see on (4.2)-(4.3) that, for $x \in[0, \delta]$, we have (dropping the dependance in $z$ ),

$$
\begin{aligned}
K_{2, R}^{+} \varphi_{1}(x)-K_{2, R}^{-} \varphi_{1}(x) \\
=\frac{1}{h^{2} \mathcal{W}\left[u_{2, R}^{-}, u_{2, R}^{+}\right]} \int_{0}^{x}\left(u_{2, R}^{-}(x) u_{2, R}^{+}(t)+u_{2, R}^{-}(t), u_{2, R}^{+}(x)\right) \varphi_{1}(t) d t \\
\quad+\frac{1}{h^{2} \mathcal{W}\left[u_{2, R}^{-}, u_{2, R}^{+}\right]} \int_{x}^{\delta}\left(u_{2, R}^{-}(t) u_{2, R}^{+}(x)+u_{2, R}^{-}(x), u_{2, R}^{+}(t)\right) \varphi_{1}(t) d t \\
\quad+\mathcal{O}\left(e^{-c^{\prime} / h}\right),
\end{aligned}
$$

with $c^{\prime}=c^{\prime}(\delta)>0$ constant, and therefore,

$$
\begin{aligned}
K_{2, R}^{+} \varphi_{1}(x)-K_{2, R}^{-} \varphi_{1}(x) & \\
= & \frac{1}{h^{2} \mathcal{W}\left[u_{2, R}^{-}, u_{2, R}^{+}\right]} \int_{0}^{\delta}\left(u_{2, R}^{-}(x) u_{2, R}^{+}(t)+u_{2, R}^{-}(t), u_{2, R}^{+}(x)\right) \varphi_{1}(t) d t \\
& +\mathcal{O}\left(e^{-c^{\prime} / h}\right) .
\end{aligned}
$$

Using also that $\mathcal{W}\left[u_{2, R}^{-}, u_{2, R}^{+}\right]=\frac{2}{\pi} h^{-\frac{2}{3}}+\mathcal{O}\left(h^{-\frac{1}{3}}\right)$ (see [FMW1, Appendix A.2], and the fact that $\varphi_{1}(x)$ is real for $x$ real, we conclude,

$$
T_{0,3}=\left(\pi h^{-\frac{4}{3}}+\mathcal{O}\left(h^{-1}\right)\right)\left\langle u_{2, R}^{-}, \varphi_{1}\right\rangle_{I_{R}^{+}}\left\langle u_{2, R}^{+}, \varphi_{1}\right\rangle_{I_{R}^{-}}+\mathcal{O}\left(e^{-c^{\prime \prime} / h}\right),
$$

(still with $c^{\prime \prime}>0$ constant). Hence, 9.7 follows by using Proposition 9.1 .

We conclude from the previous proposition and (9.1) that we have,

$$
T_{0}(z)=8 i \pi h^{-\frac{1}{3}} c_{0}^{2} a_{0}(0)^{2} f\left(h^{-\frac{2}{3}} z\right)+\mathcal{O}(1),
$$

with,

$$
f(\rho):=\left(A^{-}(\rho)+A^{+}(\rho)\right)^{2} .
$$

Then, writing,

$$
f\left(h^{-\frac{2}{3}} z\right)=f\left(h^{-\frac{2}{3}} \lambda_{0}\right)+\mathcal{O}\left(h^{-\frac{2}{3}}\right)\left(z-\lambda_{0}\right),
$$

and going back to 3.10 , we obtain,

$$
r_{0}(t, \phi, h)=4 h^{2-\frac{1}{3}} c_{0}^{2} a_{0}(0)^{2} f\left(h^{-\frac{2}{3}} \lambda_{0}\right) \int_{\gamma_{-}} \frac{e^{-i t z} g(\operatorname{Re} z)}{\left(\lambda_{0}-z\right)^{2}} d z+\mathcal{O}(h),
$$

and then, by arguments similar to those of Section 7 ,

$$
r_{0}(t, \phi, h)=4 h^{2-\frac{1}{3}} c_{0}^{2} a_{0}(0)^{2} f\left(h^{-\frac{2}{3}} \lambda_{0}\right) \int_{\gamma_{-}} \frac{e^{-i t z} g(\operatorname{Re} z)}{\left(\lambda_{0}-z\right)^{2}} d z+\mathcal{O}\left(h\langle h t\rangle^{-\infty}\right) .
$$


Finally, using (2.3) and making the change of variable $z \mapsto \lambda_{0}+h z$, we find, $r_{0}(t, \phi, h)=4 h^{\frac{2}{3}} c_{0}^{2} a_{0}(0)^{2} e^{-i t \lambda_{0}} f\left(h^{-\frac{2}{3}} \lambda_{0}\right) \int_{\gamma_{0}} \frac{e^{-i t h z} g_{0}(\operatorname{Re} z)}{z^{2}} d z+\mathcal{O}\left(h\langle h t\rangle^{-\infty}\right)$, and 2.7 is proved with,

$$
A_{0}(s)=\tau_{1}^{-\frac{1}{6}} \tau_{2}^{-\frac{1}{6}} \int_{-\infty}^{+\infty} \check{\mathrm{Ai}}\left(\tau _ { 2 } ^ { \frac { 1 } { 3 } } ( y + \frac { s } { \tau _ { 2 } } ) \mathrm { Ai } \left(\tau_{1}^{\frac{1}{3}}\left(y-\frac{s}{\tau_{1}}\right) d y .\right.\right.
$$

The fact that one also has,

$$
A_{0}(s)=\tau_{1}^{-\frac{1}{6}} \tau_{2}^{-\frac{1}{6}}\left(\tau_{1}+\tau_{2}\right)^{-\frac{1}{3}} \check{\mathrm{Ai}}\left(\left(\frac{\tau_{1}+\tau_{2}}{\tau_{1} \tau_{2}}\right)^{\frac{2}{3}} s\right)
$$

is proved in Appendix 2.

\section{Estimate on $b(\phi, h)$}

Let $\Psi_{0} \in L^{2}\left(I_{L} \cup I_{R}^{+}\right.$be the resonant state associated with $\rho_{0}$, that is the solution to $H \Psi_{0}=\rho_{0} \Psi_{0}$ normalized in such a way that,

$$
\left\langle\Psi_{0}^{\theta}, \Psi_{0}^{-\theta}\right\rangle=1
$$

where $\Psi_{0}^{ \pm \theta}:=U_{ \pm \theta} \Psi_{0}$. Then, by definition of $b(\phi, h)$, we have,

$$
b(\phi, h)=\left\langle\phi_{\theta}, \Psi_{0}^{-\theta}\right\rangle\left\langle\Psi_{0}^{\theta}, \phi_{-\theta}\right\rangle .
$$

According to [FMW1, Remark 7.1], we have,

$$
\begin{gathered}
\Psi_{0}=\left(\begin{array}{c}
C_{1} u_{1, L}^{-} l_{z=\rho_{0}}+\mathcal{O}\left(h^{\frac{2}{3}}\right) \\
\mathcal{O}\left(h^{\frac{1}{3}}\right)
\end{array}\right) \quad \text { on } I_{L} ; \\
\Psi_{0}=\left(\begin{array}{c}
(-1)^{k} C_{1} u_{1, R}^{-} l_{z=\rho_{0}}+\mathcal{O}\left(h^{\frac{2}{3}}\right) \\
\mathcal{O}\left(h^{\frac{1}{3}}\right)
\end{array}\right) \quad \text { on } I_{R}^{+},
\end{gathered}
$$

where the integer $k \geq 0$ is such that $\sin \frac{\mathcal{A}\left(\rho_{0}\right)}{h}=(-1)^{k}+\mathcal{O}\left(h^{\frac{2}{3}}\right)$, and the coefficient $C_{1}=C_{1}(h)$ is such that (10.1) is verified. We also have,

$$
\begin{gathered}
\phi=\left(\begin{array}{c}
c_{0} u_{1, L}^{-} l_{z=\lambda_{0}} \\
0
\end{array}\right) \quad \text { on } I_{L} ; \\
\phi=\left(\begin{array}{c}
(-1)^{k} c_{0} u_{1, R}^{-} l_{z=\lambda_{0}} \\
0
\end{array}\right) \text { on } I_{R}^{+} .
\end{gathered}
$$

Moreover, $\rho_{0}-\lambda_{0}=\mathcal{O}\left(h^{\frac{4}{3}}\right)$ (see [FMW1, Section2]), and thus, since $\partial_{z} u_{1, L}^{-}$ is $\mathcal{O}\left(h^{-1}\right)$ locally uniformly in $x$ (and exponentially decays at infinity), we get,

$$
\begin{gathered}
\Psi_{0}=\left(\begin{array}{c}
C_{1} u_{1, L}^{-} l_{z=\lambda_{0}} \\
0
\end{array}\right)+\mathcal{O}\left(h^{\frac{1}{3}}\right) \quad \text { on } I_{L} \\
\Psi_{0}=\left(\begin{array}{c}
(-1)^{k} C_{1} u_{1, R}^{-} l_{z=\lambda_{0}} \\
0
\end{array}\right)+\mathcal{O}\left(h^{\frac{1}{3}}\right) \text { on } I_{R}^{+} .
\end{gathered}
$$


As a consequence, we necessarily have $C_{1}= \pm c_{0}\left(1+\mathcal{O}\left(h^{\frac{1}{3}}\right)\right)$, and 2.6 follows.

\section{Appendix 1: proof of Proposition 6.1}

We only consider the case of $I_{L}$ (the one of $I_{R}^{ \pm}$being similar).

Let $v \in \mathcal{F}_{1}\left(I_{L}\right)$. We have,

$$
K_{1, L} v(x)=\mathcal{O}\left(h^{-\frac{4}{3}}\right) \int_{I_{L}}\left(u_{1, L}^{-}(x) u_{1, L}^{+}(t) \mathbf{1}_{x<t}+u_{1, L}^{-}(t) u_{1, L}^{+}(x) \mathbf{1}_{x>t}\right) v(t) d t
$$

We fix $\delta>0$ arbitrarily small, and we first suppose that $\underline{x \leq x^{*}-\delta}$. In the integral of (11.1), we decompose $I_{L}$ into three parts:

$$
I_{L}=\left(-\infty, x^{*}-\frac{\delta}{2}\right] \cup\left[x^{*}-\frac{\delta}{2},-\delta\right] \cup[-\delta, 0] .
$$

- On $\left(-\infty, x^{*}-\frac{\delta}{2}\right]$ : There, we have $u_{1, L}^{-}(x) u_{1, L}^{+}(t) \mathbf{1}_{x<t}+u_{1, L}^{-}(t) u_{1, L}^{+}(x) \mathbf{1}_{x>t}=$ $\mathcal{O}\left(h^{\frac{1}{3}} e^{-c_{0}|x-t| / h}\right)$ and $v(t)=\mathcal{O}\left(e^{-c_{1}|t| / h}\right)$, with $c_{0}, c_{1}>0$ constants. Using that $|x-t|+|t| \geq \frac{1}{2}(|x|+|t|)$, we obtain,

$h^{-\frac{4}{3}} \int_{-\infty}^{x^{*}-\frac{\delta}{2}}\left(u_{1, L}^{-}(x) u_{1, L}^{+}(t) \mathbf{1}_{x<t}+u_{1, L}^{-}(t) u_{1, L}^{+}(x) \mathbf{1}_{x>t}\right) v(t) d t=\mathcal{O}\left(h^{-1} e^{-c_{2}|x| / h}\right)$,

with $c_{2}:=\frac{1}{2} \min \left(c_{0}, c_{1}\right)$.

- On $\left(x^{*}-\frac{\delta}{2},-\delta\right]$ : There, we have $u_{1, L}^{-}(x) u_{1, L}^{+}(t) \mathbf{1}_{x<t}+u_{1, L}^{-}(t) u_{1, L}^{+}(x) \mathbf{1}_{x>t}=$ $u_{1, L}^{-}(x) u_{1, L}^{+}(t)=\mathcal{O}\left(h^{\frac{1}{3}} e^{-c_{0}|x| / h} m_{*}(t)\right)$ and $v(t)=\mathcal{O}\left(m_{*}(t)\right)$, with $c_{0}>0$ constant, and we obtain,

$h^{-\frac{4}{3}} \int_{x^{*}-\frac{\delta}{2}}^{-\delta}\left(u_{1, L}^{-}(x) u_{1, L}^{+}(t) \mathbf{1}_{x<t}+u_{1, L}^{-}(t) u_{1, L}^{+}(x) \mathbf{1}_{x>t}\right) v(t) d t=\mathcal{O}\left(h^{-1} e^{-c_{0}|x| / h}\right)$.

- On $(-\delta, 0]$ : There, we have $u_{1, L}^{-}(x) u_{1, L}^{+}(t) \mathbf{1}_{x<t}+u_{1, L}^{-}(t) u_{1, L}^{+}(x) \mathbf{1}_{x>t}=$ $u_{1, L}^{-}(x) u_{1, L}^{+}(t)=\mathcal{O}\left(h^{\frac{1}{3}} e^{-c_{0}|x| / h} m_{0}(t)\right)$ and $v(t)=\mathcal{O}\left(m_{0}(t)\right)$, with $c_{0}>0$ constant, and we obtain,

$h^{-\frac{4}{3}} \int_{-\delta}^{0}\left(u_{1, L}^{-}(x) u_{1, L}^{+}(t) \mathbf{1}_{x<t}+u_{1, L}^{-}(t) u_{1, L}^{+}(x) \mathbf{1}_{x>t}\right) v(t) d t=\mathcal{O}\left(h^{-1} e^{-c_{0}|x| / 2 h}\right)$.

Thus, on $\left(-\infty, x^{*}-\delta\right]$, we have,

$$
K_{1, L} v(x)=\mathcal{O}\left(e^{-c_{3}|x| / h}\right),
$$

with $c_{3}>0$ constant. 
Suppose now that $x^{*}-\delta \leq x \leq x^{*}$. This time we divide $I_{L}$ into,

$$
I_{L}=\left(-\infty, x^{*}-2 \delta\right] \cup\left[x^{*}-2 \delta, x^{*}\right] \cup\left[x^{*},-\delta\right] \cup[-\delta, 0] .
$$

- On $\left(-\infty, x^{*}-2 \delta\right]$ : There, we have $u_{1, L}^{-}(x) u_{1, L}^{+}(t) \mathbf{1}_{x<t}+u_{1, L}^{-}(t) u_{1, L}^{+}(x) \mathbf{1}_{x>t}=$ $u_{1, L}^{-}(t) u_{1, L}^{+}(x)=\mathcal{O}\left(e^{-c_{0} / h} m_{*}(x)\right)$ and $v(t)=\mathcal{O}\left(e^{-c_{1}|t| / h}\right)$, with $c_{0}, c_{1}>0$ constants, and we obtain, $h^{-\frac{4}{3}} \int_{-\infty}^{x^{*}-2 \delta}\left(u_{1, L}^{-}(x) u_{1, L}^{+}(t) \mathbf{1}_{x<t}+u_{1, L}^{-}(t) u_{1, L}^{+}(x) \mathbf{1}_{x>t}\right) v(t) d t=\mathcal{O}\left(m_{*}(x) e^{-c_{0} / 2 h}\right)$.

- On $\left[x^{*}-2 \delta, x^{*}\right]$ : There, we have $u_{1, L}^{-}(x) u_{1, L}^{+}(t) \mathbf{1}_{x<t}+u_{1, L}^{-}(t) u_{1, L}^{+}(x) \mathbf{1}_{x>t}=$ $\mathcal{O}\left(h^{\frac{1}{3}} m_{*}(x) m_{*}(t) e^{-c_{0}\left|\left(x^{*}-x\right)^{\frac{3}{2}}-\left(x^{*}-t\right)^{\frac{3}{2}}\right| / h}\right.$ and $v(t)=\mathcal{O}\left(m_{*}(t) e^{-c_{1}\left(x^{*}-t\right)^{\frac{3}{2}} / h}\right)$, with $c_{0}, c_{1}>0$ constants, and making the change of variable $t \mapsto x^{*}-t$, and using the notation $\widetilde{x}:=x^{*}-x$, we obtain,

$$
\begin{gathered}
h^{-\frac{4}{3}} \int_{x^{*}-2 \delta}^{x^{*}}\left(u_{1, L}^{-}(x) u_{1, L}^{+}(t) \mathbf{1}_{x<t}+u_{1, L}^{-}(t) u_{1, L}^{+}(x) \mathbf{1}_{x>t}\right) v(t) d t \\
=\mathcal{O}\left(h^{-1} m_{*}(x)\right) \int_{0}^{2 \delta} \frac{e^{-\left(c_{0}\left|t^{\frac{3}{2}}-\widetilde{x}^{\frac{3}{2}}\right|+c_{1} t^{\frac{3}{2}}\right) / h}}{\sqrt{t}} d t .
\end{gathered}
$$

Thus, using the fact that $\left|t^{\frac{3}{2}}-\widetilde{x}^{\frac{3}{2}}\right|+t^{\frac{3}{2}} \geq \frac{1}{2}\left(\widetilde{x}^{\frac{3}{2}}+t^{\frac{3}{2}}\right)$, this gives us,

$$
\begin{aligned}
h^{-\frac{4}{3}} \int_{x^{*}-2 \delta}^{x^{*}} & \left(u_{1, L}^{-}(x) u_{1, L}^{+}(t) \mathbf{1}_{x<t}+u_{1, L}^{-}(t) u_{1, L}^{+}(x) \mathbf{1}_{x>t}\right) v(t) d t \\
& =\mathcal{O}\left(h^{-1} m_{*}(x) e^{-c_{2} \widetilde{x}^{\frac{3}{2}}}\right) \int_{0}^{2 \delta} \frac{e^{-c_{2} t^{\frac{3}{2}} / h}}{\sqrt{t}} d t=\mathcal{O}\left(h^{-\frac{2}{3}} m_{*}(x) e^{-c_{2} \widetilde{x}^{\frac{3}{2}}}\right),
\end{aligned}
$$

with $c_{2}=\frac{1}{2} \min \left(c_{0}, c_{1}\right)$.

- On $\left[x^{*},-\delta\right]$ : There, we have $u_{1, L}^{-}(x) u_{1, L}^{+}(t) \mathbf{1}_{x<t}+u_{1, L}^{-}(t) u_{1, L}^{+}(x) \mathbf{1}_{x>t}=$ $u_{1, L}^{-}(x) u_{1, L}^{+}(t)=\mathcal{O}\left(h^{\frac{1}{3}} m_{*}(x) m_{*}(t) e^{-c_{0}\left(x^{*}-x\right)^{\frac{3}{2}} / h}\right.$ and $v(t)=\mathcal{O}\left(m_{*}(t)\right)$, with $c_{0}>0$ constant, and we obtain,

$$
\begin{aligned}
h^{-\frac{4}{3}} \int_{x^{*}}^{-\delta} & \left(u_{1, L}^{-}(x) u_{1, L}^{+}(t) \mathbf{1}_{x<t}+u_{1, L}^{-}(t) u_{1, L}^{+}(x) \mathbf{1}_{x>t}\right) v(t) d t \\
& =\mathcal{O}\left(h^{-1} m_{*}(x) e^{-c_{0}\left(x^{*}-x\right)^{\frac{3}{2}} / h}\right) .
\end{aligned}
$$

- On $[-\delta, 0]$ : There, we have $u_{1, L}^{-}(x) u_{1, L}^{+}(t) \mathbf{1}_{x<t}+u_{1, L}^{-}(t) u_{1, L}^{+}(x) \mathbf{1}_{x>t}=$ $u_{1, L}^{-}(x) u_{1, L}^{+}(t)=\mathcal{O}\left(h^{\frac{1}{3}} m_{*}(x) m_{0}(t) e^{-c_{0}\left(x^{*}-x\right)^{\frac{3}{2}} / h}\right.$ and $v(t)=\mathcal{O}\left(m_{0}(t)\right)$, with $c_{0}>0$ constant, and we obtain,

$$
\begin{gathered}
h^{-\frac{4}{3}} \int_{-\delta}^{0}\left(u_{1, L}^{-}(x) u_{1, L}^{+}(t) \mathbf{1}_{x<t}+u_{1, L}^{-}(t) u_{1, L}^{+}(x) \mathbf{1}_{x>t}\right) v(t) d t \\
=\mathcal{O}\left(h^{-1} m_{*}(x) e^{-c_{0}\left(x^{*}-x\right)^{\frac{3}{2}} / h}\right) .
\end{gathered}
$$


Thus, on $\left(x^{*}-\delta, x^{*}\right]$, we have,

$$
K_{1, L} v(x)=\mathcal{O}\left(h^{-1} m_{*}(x) e^{-c_{3}\left(x^{*}-x\right)^{\frac{3}{2}} / h}\right),
$$

with $c_{3}>0$ constant.

Then we consider the case $x^{*} \leq x \leq-\delta$. We divide $I_{L}$ into,

$$
I_{L}=\left(-\infty, x^{*}-\delta\right] \cup\left[x^{*}-\delta, x^{*}\right] \cup\left[x^{*}, 0\right] .
$$

Arguing as before, we find,

$$
\begin{aligned}
h^{-\frac{4}{3}} \int_{-\infty}^{x^{*}-\delta} & \left(u_{1, L}^{-}(x) u_{1, L}^{+}(t) \mathbf{1}_{x<t}+u_{1, L}^{-}(t) u_{1, L}^{+}(x) \mathbf{1}_{x>t}\right) v(t) d t \\
= & \mathcal{O}\left(m_{*}(x) e^{-c_{0} / h}\right) \\
h^{-\frac{4}{3}} \int_{x^{*}-\delta}^{x^{*}} & \left(u_{1, L}^{-}(x) u_{1, L}^{+}(t) \mathbf{1}_{x<t}+u_{1, L}^{-}(t) u_{1, L}^{+}(x) \mathbf{1}_{x>t}\right) v(t) d t \\
& =\mathcal{O}\left(m_{*}(x) h^{-\frac{2}{3}}\right) \\
h^{-\frac{4}{3}} \int_{x^{*}}^{0} & \left(u_{1, L}^{-}(x) u_{1, L}^{+}(t) \mathbf{1}_{x<t}+u_{1, L}^{-}(t) u_{1, L}^{+}(x) \mathbf{1}_{x>t}\right) v(t) d t \\
= & \mathcal{O}\left(m_{*}(x) h^{-1}\right),
\end{aligned}
$$

and thus, on $\left[x^{*},-\delta\right]$, we have,

$$
K_{1, L} v(x)=\mathcal{O}\left(h^{-1} m_{*}(x)\right) .
$$

Finally, in the case $-\delta \leq x \leq 0$, dividingagain $I_{L}$ into,

$$
I_{L}=\left(-\infty, x^{*}-\delta\right] \cup\left[x^{*}-\delta, x^{*}\right] \cup\left[x^{*}, 0\right],
$$

we find in the same way,

$$
K_{1, L} v(x)=\mathcal{O}\left(h^{-1} m_{0}(x)\right) .
$$

We also see that the same estimates hold for the derivatives $\left(h D_{x}\right)^{k} K_{1, L} v(x)$, and thus we have proved,

$$
K_{1, L}\left(\mathcal{F}_{1}\left(I_{L}\right)\right) \subset h^{-1} \mathcal{F}_{1}\left(I_{L}\right) .
$$

Concerning $K_{1, L}\left(\mathcal{F}_{2}\left(I_{L}\right)\right)$, that is, if $v \in \mathcal{F}_{2}\left(I_{L}\right)$, the same decompositions as before give exponentially small terms only, multiplied by $m_{*}(x) m_{0}(x)$, except those for $t$ close to 0 . For these last ones, the previous arguments permit us to estimate them by,

$$
\mathcal{O}\left(h^{-1}\right) m_{*}(x) m_{0}(x) \alpha(x) \int_{0}^{\delta} \frac{e^{-t^{\frac{3}{2}} / h}}{\sqrt{t}} d t=\mathcal{O}\left(h^{-\frac{2}{3}}\right) m_{*}(x) m_{0}(x) \alpha(x),
$$


where we have used the notation $\alpha(x):=e^{-c\left(x^{*}-x\right)^{\frac{3}{2}} / h}$ ) (with $c>0$ constant) when $x \leq x^{*}$, and $\alpha(x):=1$ when $x \geq x^{*}$. This proves that,

$$
K_{1, L}\left(\mathcal{F}_{2}\left(I_{L}\right)\right) \subset h^{-2 / 3} \mathcal{F}_{1}\left(I_{L}\right) .
$$

The estimate on $K_{2, L}\left(\mathcal{F}_{1}\left(I_{L}\right)\right)$ follows essentially in the same way, except for the behaviour near $x=x^{*}$. Take $v \in \mathcal{F}_{1}\left(I_{L}\right)$, and first consider $K_{2, L}(v)(x)$ for $x^{*}-\delta \leq x \leq x^{*}$. One has,

$$
\begin{aligned}
K_{2, L}(v)(x)= & \mathcal{O}\left(h^{-1}\right) \int_{x^{*}-\delta}^{x^{*}} e^{-c_{0}\left(|t-x|+\left|t-x^{*}\right|^{\frac{3}{2}}\right) / h} m_{*}(t) d t \\
& +\mathcal{O}\left(h^{-1}\right) \int_{x^{*}}^{x^{*}+\delta} e^{-c_{0}|t-x| / h} m_{*}(t) d t+\mathcal{O}\left(e^{-c_{0} / h}\right),
\end{aligned}
$$

with $c_{0}>0$. Making the change of variable $t \mapsto x^{*}-t$, and setting $\widetilde{x}:=$ $x^{*}-x$, we obtain,

$$
\begin{aligned}
K_{2, L}(v)(x)= & \mathcal{O}\left(h^{-1}\right) \int_{0}^{\delta} e^{-c_{0}\left(|\widetilde{x}-t|+t^{\frac{3}{2}}\right) / h} t^{-\frac{1}{4}} d t \\
& +\mathcal{O}\left(h^{-1}\right) \int_{-\delta}^{0} e^{-c_{0}(\widetilde{x}-t) / h}|t|^{-\frac{1}{4}} d t+\mathcal{O}\left(e^{-c_{0} / h}\right),
\end{aligned}
$$

and thus, using that $\left|t^{\frac{3}{2}}-\widetilde{x}^{\frac{3}{2}}\right| \leq \frac{1}{2}|\widetilde{x}-t|$ for $t, \widetilde{x} \in[0, \delta], \delta$ small enough, we deduce,

$$
\begin{aligned}
K_{2, L}(v)(x)= & \mathcal{O}\left(h^{-1}\right) e^{-c_{0} \widetilde{x}^{\frac{3}{2}} / h} \int_{0}^{\delta} e^{-c_{0}|\widetilde{x}-t| / 2 h} t^{-\frac{1}{4}} d t \\
& +\mathcal{O}\left(h^{-1}\right) \int_{0}^{\delta} e^{-c_{0}(\widetilde{x}+t) / h} t^{-\frac{1}{4}} d t+\mathcal{O}\left(e^{-c_{0} / h}\right),
\end{aligned}
$$

Making the change of variable $t \mapsto h t$, this gives us,

$$
\begin{aligned}
K_{2, L}(v)(x)= & \mathcal{O}\left(h^{-\frac{1}{4}}\right) e^{-c_{0} \widetilde{x}^{\frac{3}{2}} / h} \int_{0}^{\delta / h} e^{-c_{0}\left(h^{-1} \widetilde{x}-t\right) / 2} t^{-\frac{1}{4}} d t \\
& +\mathcal{O}\left(h^{-\frac{1}{4}}\right) \int_{0}^{\delta / h} e^{-c_{0}\left(h^{-1} \widetilde{x}+t\right)} t^{-\frac{1}{4}} d t+\mathcal{O}\left(e^{-c_{0} / h}\right),
\end{aligned}
$$

and, cutting the first integral into $\int_{0}^{1}+\int_{1}^{\widetilde{x} / h}+\int_{\widetilde{x} / h}^{\delta / h}$ in the case $\widetilde{x} \geq h$, and into $\int_{0}^{1}+\int_{1}^{\delta / h}$ in the case $0 \leq \widetilde{x} \leq h$, we obtain,

$$
K_{2, L}(v)(x)=\mathcal{O}\left(h^{-\frac{1}{4}}\right) e^{-c_{0} \widetilde{x}^{\frac{3}{2}} / h}+\mathcal{O}\left(h^{-\frac{1}{4}}\right) e^{-c_{0} \tilde{x} / h}
$$

and therefore (since $0 \leq \widetilde{x}^{\frac{3}{2}} \leq \widetilde{x}$ ), for $x^{*}-\delta \leq x \leq x^{*}$,

$$
K_{2, L}(v)(x)=\mathcal{O}\left(h^{-\frac{1}{4}}\right) e^{-c_{0} \widetilde{x}^{\frac{3}{2}} / h}=\mathcal{O}\left(h^{-\frac{1}{4}}\right) e^{-c_{0}\left(x^{*}-x\right)^{\frac{3}{2}} / h} .
$$

When $x^{*} \leq x \leq x^{*}+\delta$, the same computations lead to

$$
K_{2, L}(v)(x)=\mathcal{O}\left(h^{-\frac{1}{4}}\right) .
$$


Then, when $x^{*}+\delta \leq x \leq-\delta$, we can write,

$$
K_{2, L}(v)(x)=\mathcal{O}\left(h^{-1}\right) e^{-|x|^{\frac{3}{2}} / h} \int_{0}^{\delta} e^{t^{\frac{3}{2}} / h} t^{-\frac{1}{2}} d t+\mathcal{O}(1),
$$

and the change of variable $t \mapsto(h t)^{\frac{2}{3}}$ gives us,

$$
K_{2, L}(v)(x)=\mathcal{O}\left(h^{-\frac{2}{3}}\right) e^{-|x|^{\frac{3}{2}} / h} \int_{0}^{\delta^{\frac{3}{2}} / h} \frac{e^{t}}{t^{\frac{2}{3}}} d t+\mathcal{O}(1)=\mathcal{O}\left(h^{-\frac{2}{3}}\right) .
$$

Finally, for $-\delta \leq x \leq 0$, the same kind of computations lead to,

$$
K_{2, L}(v)(x)=\mathcal{O}\left(h^{-\frac{2}{3}}\right) m_{0}(x) .
$$

Since in addition $h^{-\frac{1}{4}} \leq h^{-\frac{2}{3}} m_{*}(x)$ on $\left[x^{*}-\delta, x^{*}+\delta\right]$, the required result on $K_{2, L}\left(\mathcal{F}_{1}\left(I_{L}\right)\right)$ follows from $11.2-11.5$

The estimate on $K_{2, L}\left(\mathcal{F}_{2}\left(I_{L}\right)\right)$ follows along the same lines, together with the results on $I_{R}^{ \pm}$.

\section{Appendix 2: PRoOF of 9.12}

For any tempered function $f=f(x)$ on $\mathbb{R}$, we denote by $\hat{f}$ its Fourier transform defined by,

$$
\hat{f}(\xi):=\int_{-\infty}^{+\infty} e^{-i x \xi} f(x) d x
$$

and, for $\alpha>0$ constant and $x \in \mathbb{R}$, we set,

$$
f_{\alpha}(x):=\operatorname{Ai}(\alpha x) .
$$

By definition we have $\hat{\mathrm{Ai}}(\xi)=e^{i \xi^{3} / 3}$, and thus $\hat{f}_{\alpha}(\xi)=\alpha^{-1} e^{i \alpha^{-3} \xi^{3} / 3}$.

Then, we see on (9.11) that we have,

$$
A_{0}(s)=g(x):=\tau_{1}^{-\frac{1}{6}} \tau_{2}^{-\frac{1}{6}}\left(f_{\alpha} * f_{\beta}\right)(x)
$$

with,

$$
\alpha:=\tau_{1}^{\frac{1}{3}} \quad ; \quad \beta:=\tau_{2}^{\frac{1}{3}} \quad ; \quad x:=-\left(\frac{\tau_{1}+\tau_{2}}{\tau_{1} \tau_{2}}\right) s,
$$

and where $*$ stands for the standard convolution of functions. As a consequence, using that $\left(f_{\alpha} * f_{\beta}\right)=\hat{f}_{\alpha} \hat{f}_{\beta}$, we obtain,

$$
\begin{aligned}
\hat{g}(\xi) & =\tau_{1}^{-\frac{1}{6}} \tau_{2}^{-\frac{1}{6}} \hat{f}_{\alpha}(\xi) \hat{f}_{\beta}(\xi)=\tau_{1}^{-\frac{1}{6}} \tau_{2}^{-\frac{1}{6}} \alpha^{-1} \beta^{-1} e^{i\left(\alpha^{-3}+\beta^{-3}\right) \xi^{3} / 3} \\
& =\tau_{1}^{-\frac{1}{6}} \tau_{2}^{-\frac{1}{6}} \gamma \alpha^{-1} \beta^{-1} \hat{f}_{\gamma}(\xi),
\end{aligned}
$$

with,

$$
\gamma:=\left(\alpha^{-3}+\beta^{-3}\right)^{-\frac{1}{3}}=\left(\frac{\tau_{1} \tau_{2}}{\tau_{1}+\tau_{2}}\right)^{\frac{1}{3}} .
$$


Hence,

$$
g=\tau_{1}^{-\frac{1}{6}} \tau_{2}^{-\frac{1}{6}} \gamma \alpha^{-1} \beta^{-1} f_{\gamma}=\tau_{1}^{-\frac{1}{6}} \tau_{2}^{-\frac{1}{6}}\left(\tau_{1}+\tau_{2}\right)^{-\frac{1}{3}} f_{\gamma},
$$

and 9.12$)$ follows.

\section{REFERENCES}

[BCD] Briet, P., Combes, J.-M., Duclos, P., On the location of resonances for Schrödinger operators in the semiclassical limit. I. Resonances free domains, J. Math. Anal. Appl. 126 (1987), no. 1, 90-99.

[BrMa] Briet, P., Martinez, A., Estimate on the molecular dynamics for the predissociation process, J. Spect. Theory 7 (2017), no. 2, 487-517.

[BrMa3] Briet, P., Martinez, A., Molecular dynamics at energy level crossing II: the physical case, In progress.

[CGH] Cattaneo, L., Graf, G. M., Hunziker, W., A general resonance theory based on Mourre's inequality, Ann. H. Poincaré 7, 2006 No. 1, 583-601.

[CoSo] Costin, O., Soffer, A., Resonance theory for Shrödinger operators, Comm. Math. Phys. 224, 133-152, 2001.

[DuMe] Duclos, P., Meller, B., A simple model for predissociation, Mathematical results in quantum mechanics (Blossin, 1993), Oper. Theory Adv. Appl., 10, Birkaüser, Basel, 1994.

[FMW1] Fujiié, S., Martinez, A., Watanebe, T. Molecular predissociation resonances near an energy-level crossing I: Elliptic interaction, J.Differential Equations 260 (2016), no. 5, 4051-4085.

[FMW2] Fujiié, S.; Martinez, A.; Watanabe, T. Molecular predissociation resonances near an energy-level crossing II: Vector field interaction. J. Differential Equations 262 (2017), no. $12,5880-5895$.

[GrMa] Grigis, A., Martinez, A. Resonance widths for the molecular predissociation, Analysis \& PDE 7-5 (2014), 1027-1055. DOI 10.2140/apde.2014.7.1027

[HeMa] Helffer, B., Martinez, A., Comparaison entre les diverses notions de résonances, Helv. Phys. Acta, Vol.60 (1987),no.8, pp.992-1003.

[HeRo] Helffer, B., Robert, D., Puits de potentiel généralisé et asymptotique semiclassique, Ann. Inst. H. Poincaré, Phys. Theor. 41, 1984, No. 3, 291-331.

[HeSj1] Helffer, B., Sjöstrand, J., Multiple Wells in the Semiclassical Limit I, Comm. in P.D.E. 9(4) (1984), pp.337-408.

[HeSj2] Helffer, B., Sjöstrand, J., Résonances en limite semi-classique, Bull. Soc. Math. France 114, Nos. 24-25 (1986).

[Her] Herbst, I., Exponential decay in the Stark effect, Comm. Math. Phys., 75, 197-205, 1980.

[Hu1] Hunziker, W., Distortion analyticity and molecular resonance curves, Ann. Inst. H. Poincaré Phys. Théor. 45 (1986), no. 4, pp. 339-358.

[Hu2] Hunziker, W., Resonances, metastable states and exponential decay laws in perturbation theory, Comm. Math. Phys. 132, 177-182, 1990.

[JeNe] Jensen, A., Nenciu, G., The Fermi golden rule and its form at thresholds in odd dimensions, Comm. Math. Phys. 261, 693-727, 2006.

[Kl] Klein, M., On the mathematical theory of predissociation, Annals of Physics, Vol. 178, No. 1, 48-73 (1987).

[Kr] Kronig, L. de R., Z. Phys. 50, 247 (1928)

[KMSW] Klein, M., Martinez, A., Seiler, R., Wang, X.P. On the Born-Oppenheimer Expansion for Polyatomic Molecules, Comm. Math. Physics 143, (1992), no.3, pp. 607-639

[La] Landau, L., Phys. Z. Sowjetunion 1 (1932), 89; 2 (1932), 46.

[LeSu] Lee S., Sun, H., Widths and positions of isolated resonances in the pre dissociation of SH: Quantal treatments, Bull. Korean Chem. Soc. 2001, Vol. 22, No.2, 210-212.

[Ma1] Martinez, A., Resonance free domains for non globally analytic potentials, Ann. Henri Poincaré 4, 739-756 (2002), Erratum: Ann. Henri Poincaré 8 (2007), 1425-1431 
[Ma2] Martinez, A., An Introduction to Semiclassical and Microlocal Analysis, UTX Series, Springer-Verlag New-York (2002).

[MaSo] Martinez, M., Sordoni, V., Twisted pseudodifferential calculus and application to the quantum evolution of molecules, Memoirs of the AMS, No. 936 (2009).

[NSZ] Nakamura, S., Stefanov, P., Zworski, M., Resonance expansion of propagators in the presence of potential barriers, J. Funct. Anal. 205, 2003 No. 1, 180-205.

[ReSi] Reed, M., Simon, B., Methods of Modern Mathematical Physics IV: Analysis of Operators, Academic Press INC., New York, 1978.

[Si] Simon, B., Semiclassical analysis if low lying eigenvalues I. Non-degenerate minima: Asymptotic expansions, Annales Inst. H. Poincaré, Section A, 38, No. 3, 1983, 295-308.

[St] Stückelberg, E. C. G., Helv. Phys. Acta 5, 370 (1932).

[Ze] Zener, C., Proc. R. Soc. London Ser. A 137, 696 (1932). 